\title{
Weakly Nonlinear Waves on Surface of Ideal Finite Depth Fluid
}

\author{
V.Zakharov
}

\section{Introduction}

The waves on a surface of an incompressible fluid are one of the most common phenomena we observe in our life. Since the begining of the last century the surface waves became the subject of intense analytical study. The theory of surface waves attracted the attention of many first class mathematicians. Boussinesq, Stokes and Airy are just the most famous names among them.

The linear theory of surface waves of infinitely small amplitude was mostly accomplished hundred years ago. Some achievments of these efforts were seminal to the Mathematical Physics as whole. For example, the Method of Stationary Phase was discovered in connection with the problem of surface waves.

In spite of the fact that the mathematical aspect of wave propogation is one of the classical subjects of Mathematical Physics, the theory of surface waves for many decades was an isolated island, just weakly connected with the main continent, the theory of sound and the theory of electromagnetic waves. One of the reasons for this was a dispersion. In a contrary to the light and to the sound, the waves on the surface of an incompressible fluid are strongly dispersive. Their phase velocity depends essentially on a wave number. Another reason was a beleif that the theory of surface waves is not a "normal" subject of pure mathematics. The basic equations describing waves on a surface of an ideal fluid in their classical formulation are neither ordinar no partial differential equations. They look like an "orphan" in a society of normal PDE equations, like the Maxwell equations or linearizes Navier-Stokes equations describing the "ordinary" waves.

Nevertheless, the theory of surface waves became a cradle of the modern theory of waves in nonlinear dispersive media. It was Stokes who formulated the concept of a "progressive stationary wave" and calculated the nonlinear correction to dispersion relation. Another fundamental concept of modern nonlinear physiscs, the soliton, was also born in the theory of surface waves.

The isolation of the theory of surface waves was broken in fifties and sixties of this century. The fast development of plasma and solid state physics showed that a strong dispersion is a common thing for waves in real media, and non-dispersive sound and light waves are just very special exclusions in the world of waves, which mostly are strongly 
dispersive. In the last three decades the the surface waves became a subject of intense study.

In 1966 the author of this article have found that the waves on a surface of ideal fluid in mathematical sense are Hamiltonian systems and their theory can be treated as a chapter in a general theory of dispersive waves. This fact made possible to establish numerous parallels between the theory of surface waves and theories of waves of different type in plasmas and nonlinear optics. Due to "translation" of basic statements of surface wave theory to a "Hamiltonian language", it became possible to extend an essential part of the results obtained in this field to the waves of quite different types, like spin waves in ferromagnetics, for example.

¿From the mathematical point of view the system of surface waves is a Hamiltonian system of infinite number degrees of freedom. In reality, the number of degrees of freedom is finite but very large. In a typical situation it could reach the order of magnitude about $10^{8}$ or more. Hence the problem of statistical description of the surface waves is very urgent. No doubt that a real behaviour of a sea surface is a stochastic process that has many common features with a turbulence in an incomperrible fluid at the large Reinolds number, $R \simeq 10^{5}$ or more.

In a contrary to the turbulence in incompressible fluid, the turbulence of surface waves is weak. It means that the wave taking part in this turbulence is "almost linear". Foe the waves on deep water the measure of their nonlinearity is a parameter $\mu \simeq(k a)^{2}$, where $k$ is a characteristic wave number, and $a$ is a typical wave amplitude. In a real situation this parameter is small, $\mu \simeq 10^{-3}$ or even less. The physical reason for this fact is obvious, that is existense of another small parameter, $\epsilon \sim \rho_{a} / \rho_{w} \sim 10^{-3}$, where $\rho_{a}, \rho_{w}$ are air and water densities. A relative wave amplitude is small because waves on a surface of a heavy fluid (water) are excited by the motion of a very light substance (air). This consideration is a qualitative one. It is a very sophysticated problem to find of the analytical connection between $\mu$ and $\epsilon$, because of a strong turbulence in the wind-driven sea waves.

Anyway, the parameter $\mu$ is small. This fact makes possible to develop close and selfsustained description of surface wave turbulence. On deep water it is described by the kinetic equation for spectral density of wave action, derived first by K.Hasselmann (Hasselmann, 1962). It was a great achievment of the theory of surface waves. It's correctness was confirmed by numerous comparisons with experiment, and succesfull development of effective models for wave prediction (WAM model, see Komen et al, 1994). However, after thirty five satisfactory years, we can see now the limitations of this approach. For instanse, we can not find the space-time correlation function for surface waves. Moreover, it can be applied only to waves on deep water.

Meanwhile, the experimentally established weakness of surface wave interactions makes possible to develop approximate dynamic models of nonlinear surface wave interaction, much more convinient for an analytical stady than the initial exact Hamiltonian equation. For deep water such model was found in 1968 (Zakharov, 1968). Since this time it was used by many authors for analytical and numerical study of nonlinear surface waves. This approximate dynamic model is a solid foundation for statistical description of surface waves. 
It was done in the works of Zakharov and Filonenko (Zakharov, Filonenko, 1966), and Saffmann, Lake and Yean (Saffmann et al, 1980). It was established recently (Dyachenko, Lvov, 1996) that the kinetic equation found in this way is identical to the equation initially derived by K.Hasselmann.

The capacities of the approximate analytical model derived in 1968 are not implemented in a proper degree to the statistical description of surface waves so far. It will be a subject of future publication.

The purpose of this article is to develop the approximate dynamical model for description of weakly nonlinear surface waves for the case of fluid of finite depth. We realize that construction of this model is a first and a very serious step towards developing of a proper model for statistical description of waves on surface of finite-depth water. The situation in this case is much more complicated than on deep water. Besides of the parameter $\mu$, we have now a parameter $\delta=k h$, where $h$ is the fluid depth. The case $k h \rightarrow 0$ (very shallow water) is espesially interesting. It is known since the time of Stokes that progressive stationary waves are close to linear harmonics if only a very restrictive condition holds:

$$
\mu \ll \delta^{4}
$$

We will show ( not in this article) that for a broad in $k$-space spectra the Stokes condition can be released up to the level

$$
\mu \ll \delta^{3}
$$

In reality the parameter $\delta$ is not too small. For instanse, if $L / h \sim 30$ ( $L$ is a wave length), $\delta \simeq 0.2$, and the condition (1.2) still holds if $\mu \simeq 10^{-3}$. This development of the weakly nonlinear dynamical model makes sense even for waves on a very shallow water.

The basic point for construction of a proper dynamical model on deep water is an exclusion of quadratic nonlinearities in the dynamical equation by a multiscale expansion or by a canonical transformation. Theoretically speaking, the same technique can be emplemented in the case of finite depth. However, we have to stress that two new curcumstances, entirely pertaining to the case of finite depth, could play a very important role in any future statistical theory.

The first one is a dissipation of wave energy that takes place in the boundary layer near the sea bottom. Itis important to underline that this is not only a relatively small contribution to the linear decrement of a wave decay. Much more important effect is the dissipation of energy by the forced, almost resonant beabing. This is nonlinear, but "non-Hamiltonian" effect, quite similiar to a well-known "non-linear Landau damping" of Langmuir plasma waves. Even in the case of a moderate depth, $k h \simeq 1$, this damping can produce non-Hamiltonian non-linear interactions overriding the classical four-wave processes.

The second effect takes place if only the parameter $\delta$ is small enough, $\delta \ll 1$. In this case the leading terms in the coefficient four-wave interaction, and in higher-order coefficients as well, exactly cancelled. This cancellation is an quite nontrivial and deeply 
hidden effect. It can be explained in a following way. In the case of a very shallow water nonlinear interactions of surface waves are described approximately by the well-known Kadomtsev - Petviashvili (KP-2) equation. However, this equation is an exactly integrable Hamiltonian system. In practice this integrability is realizes by holding an infinite number of very unexpected identities, which can not be easely checked even in the most simple cases.

Both mentioned curcumstances will play a very important role in a future statistical theory of gravity waves on the surface of a finite-depth fluid.

\section{Hamiltonian formalism}

We study waves on the surface of an ideal fluid in an infinite basin of constant depth $h$. Let $z$ be the vertical coordinate. The interpurbed fluid surface is posed at $z=0$, the bottom at $z=-h$. Coordinates in the horizontal plane are $x, y, r=(x, y)$, the displacement of the surface is $\eta(r, t)=\eta(x, y, t)$. The density of the fluid $\rho_{w}=1$. The gravity acceleration $g$ is directed downward, the surface tension coefficient is $\sigma$. Further we will apply the developed theory for the description of a real situation when the upper half-space $z>\eta(\vec{r}, t)$ is filled with air. We will assume that the air density $\rho_{a}$ is small, thus $\epsilon=\rho_{a} / \rho_{w}$ is a small parameter $\epsilon \ll 1$.

We will suppose that the fluid is incompressible, $\operatorname{div} V=0$, and that the velocity field $V$ is potential one:

$$
V=\nabla \Phi
$$

Let us assume that:

$$
\Psi(r, t)=\left.\Phi\right|_{z=\eta(r, t)},
$$

where the potential $\Phi$ satisfies the Laplace equation

$$
\Delta \Phi=0
$$

under the boundary conditions:

$$
\left.\Phi\right|_{z=\eta}=\left.\Psi(r, t) \quad \Phi_{z}\right|_{z=-h}=0 .
$$

The boundary problem $(2.3),(2.4)$ has a unique solution. Hence we can find the velocity field $V(\vec{r}, t)$ if $\eta(r, t)$ and $\Psi(r, t)$ are given. The total energy of the fluid, $H=T+U$, has the following expressions for kinetic and potential energies:

$$
\begin{gathered}
T=\frac{1}{2} \int d r \int_{-h}^{\eta}(\nabla \Phi)^{2} d z \\
U=\frac{1}{2} g \int \eta^{2} d r+\sigma \int\left(\sqrt{1+\left(\nabla_{\perp} \eta\right)^{2}}-1\right) d r .
\end{gathered}
$$


Traditionally, the dynamics of a free surface are described by imposing at $z=\eta$ of two nonlinear boundary conditions (see, for instance, Stokes):

$$
\begin{gathered}
\frac{\partial \eta}{\partial t}=+\left(-\nabla_{\perp} \Phi \nabla_{\perp} \eta+\frac{\partial \Phi}{\partial z}\right) \\
\left.\frac{\partial \Phi}{\partial t}\right|_{z=\eta}=-\left.\frac{1}{2}\left(\left(\nabla_{\perp} \Phi\right)^{2}+\left(\frac{\partial \Phi}{\partial z}\right)^{2}\right)\right|_{z=\eta}-g \eta+\sigma \nabla_{\perp} \frac{\nabla_{\perp} \eta}{\sqrt{1+\nabla_{\perp} \eta^{2}}}
\end{gathered}
$$

where

$$
\nabla_{\perp}=\vec{i} \frac{\partial}{\partial x}+\vec{j} \frac{\partial}{\partial y}
$$

The system (2.7), (2.8) is neither a system of PDE's, no even a "dynamical system". It can be turned to a dynamic system by using the identity

$$
\left.\frac{\partial \Phi}{\partial t}\right|_{z=\eta}=\frac{\partial \Psi}{\partial t}-\left.\frac{\partial \Phi}{\partial z}\right|_{z=\eta} \frac{\partial \eta}{\partial t} .
$$

Combining (2.9) and (2.7) we obtain:

$$
\begin{aligned}
\frac{\partial \Psi}{\partial t} & =\left.\left(-\frac{1}{2}\left(\nabla_{\perp} \Phi\right)^{2}+\frac{1}{2}\left(\frac{\partial \Phi}{\partial z}\right)^{2}-\frac{\partial \Phi}{\partial z} \nabla_{\perp} \Phi \nabla_{\perp}\right)\right|_{z=\eta}- \\
& -g \eta+\sigma \nabla_{\perp} \frac{\nabla_{\perp} \eta}{\sqrt{1+\left(\nabla_{\perp} \eta\right)^{2}}} .
\end{aligned}
$$

The following fact is of basic importance:

Theorem 2.1

The system (2.7), (2.10) is a Hamiltonian system:

$$
\begin{aligned}
\frac{\partial \eta}{\partial t} & =\frac{\delta H}{\delta \Psi} \\
\frac{\partial \Psi}{\partial t} & =-\frac{\delta H}{\delta \eta} .
\end{aligned}
$$

The relations (2.11), (2.12) were found by the author of this article in 1966 (Zakharov, 1966). The complete proof of Theorem 2.1 was published in 1968 (Zakharov, 1968). The original proof was relatively complicated. Later on, it was essetially simplified (Zakharov, Kuznetsov, 1986). Let us reproduce this simplified proof.

Proof 
The dynamics of an incompressible fluid must realize the minimization of action $S=\int L d t$

$$
\delta S=0
$$

where

$$
\begin{aligned}
L & =T-U+\int \Phi d i v V d z d r+\int \Psi\left(\frac{\partial \eta}{\partial t}+V_{\perp} \nabla \eta-V_{z}\right) d r \\
T & =\frac{1}{2} \int d r \int_{-\eta}^{r} V^{2} d z
\end{aligned}
$$

and $U$ is given by $(2.6)$.

The last two terms in (2.14) are the "Largangian factors". We have added them to take into account the condition of incompressibility div $V=0$, and the "kinematic" boundary condition (2.7). Using Green's formula we can transform

$$
\begin{aligned}
\int \Phi d i v V d z d r & =-\int\left(V \nabla_{\perp} \Phi\right) d z d r+\left.\int \Phi\right|_{z=\eta} V_{n} d s= \\
& =\left.\int \Phi\right|_{z=\eta}\left(V_{z}-V_{\perp} \nabla_{\perp} \eta\right) d r
\end{aligned}
$$

In virtue of (2.13), we have:

$$
\frac{\delta L}{\delta V}=0
$$

Putting in $(2.17)-h<z<\eta$ we can obtain:

$$
V=\nabla \Phi
$$

The condition (2.17) on the surface $z=\eta$ gives:

$$
\left.\Phi\right|_{z=\eta}=\Psi
$$

Taking into account (2.18) and (2.19) we can transform the Lagrangian $L$ to the form:

$$
L=\int \Psi \frac{\partial \eta}{\partial t} d r-H
$$

Variation of $L$ with respect to $\Psi$ and $\eta$ gives (2.11), (2.12).

Apparently this proof can be generalized for the case of a bottom as an arbitrary function of the coordinates:

$$
h \rightarrow h(r)
$$


For the Hamiltonian equations (2.11), (2.12) in a full 3-dimensional case one cannot express the kinetic energy $T$ in terms of canonical variables explicitly. In two-dimensional case one can realize the conformal transformation of the domain $-h<z<\eta$ to the stripe $-h<z<0$. This transformation induces the canonical transformation from the initial variables $\eta(x, t), \Psi(x, t)$ to new variables $\eta(u, t), \xi(u, t)$. Here $\eta=\eta(u, t)$ is the displacement of the surface in the conformal coordinates, $\xi$ are conjugated variables. The kinetic energy $T$ can be expressed explicitly in terms of $\eta, \xi$ (Dyachenko, Lvov, Zakharov, 1995). In a 3-D case one has to use the approximated expressions for $H$. There are two basic approximated methods for calculation of $H$. Both of them have roots in works of nineteen-century classics. The approximation of "small angles" referres to Stokes (Stokes, 1847), while the approximation of "shallow water" was invented by Airy (Airy, 1845).

\section{Approximation of Small Angles}

The boundary problem (2.2)-(2.4) can be solved explicitly for the flat surface $\eta=0$. In this case:

$$
\Phi(r, z)=\frac{1}{2 \pi} \int \Psi(k) \frac{\cos h k(z+h)}{\cos h k h} e^{i k r} d k .
$$

Here $\Psi(k)$ is the Fourier transform of $\Psi(r)$ :

$$
\Psi(k)=\frac{1}{2 \pi} \int \Psi(r) e^{-i k r} d r
$$

The kinetic energy is now:

$$
T_{0}=\frac{1}{2} \int k \tanh (k h)\left|\Psi_{k}\right|^{2} d k .
$$

For the "almost flat" surface, when

$$
\left|\nabla_{\perp} \eta\right| \ll 1
$$

one can solve the boundary problem (2.2)-(2.4) in form of a series on powers of $\nabla_{\perp} \eta$ as well. It is convinient to make the expansion for $T$ in terms of Fourier transforms

$$
T=\sum_{n=1}^{\infty} T_{n}
$$

where $T_{0}$ is given by (3.3), and $T_{n}$ is

$$
\begin{aligned}
T_{n} & =\frac{1}{2(2 \pi)^{n}} \int L^{(n)}\left(k_{1}, k_{2}, k_{3} \ldots k_{2+n}\right) \Psi_{k_{1}} \Psi_{k_{2}} \eta_{k_{3}} \ldots \eta_{k_{2}+n} \times \\
& \times \delta\left(k_{1}+k_{2}+\cdots+k_{n+2}\right) d k_{1} \ldots d k_{n+2} .
\end{aligned}
$$




$$
\begin{aligned}
L^{(1)}\left(k_{1}, k_{2}, k_{3}\right)= & -\left(k_{1} k_{2}\right)-\left|k_{1} k_{2}\right| \tanh \left(k_{1} h\right) \tanh \left(k_{2} h\right) \\
L^{(2)}\left(k_{1}, k_{2}, k_{3}, k_{4}\right)= & \frac{1}{4}\left|k_{1}\right|\left|k_{2}\right| \tanh \left(k_{1} h\right) \tanh \left(k_{2} h\right) \times \\
& \times\left\{-\frac{2\left|k_{1}\right|}{\tanh \left(k_{1} h\right)}-\frac{2\left|k_{2}\right|}{\tanh \left(k_{2} h\right)}+\left|k_{1}+k_{3}\right| \tanh \left(\left|k_{1}+k_{3}\right| h\right)+\right. \\
& +\left|k_{2}+k_{3}\right| \tanh \left(\left|k_{2}+k_{3}\right| h\right)+\left|k_{1}+k_{4}\right| \tanh \left(\left|k_{1}+k_{4}\right| h\right)+ \\
& \left.+\left|k_{2}+k_{4}\right| \tanh \left(\left|k_{2}+k_{4}\right| h\right)\right\}
\end{aligned}
$$

On deep water, when $k h \rightarrow \infty$, expressions (3.7), (3.8) simplify to

$$
\begin{aligned}
& L^{(1)}=-\left(k_{1} k_{2}\right)-\left|k_{1}\right|\left|k_{2}\right| \\
& L^{(2)}=\frac{1}{4}\left|k_{1}\right|\left|k_{2}\right|\left\{-2\left|k_{1}\right|-2\left|k_{2}\right|+\left|k_{1}+k_{3}\right|+\left|k_{1}+k_{4}\right|+\left|k_{2}+k_{3}\right|+\left|k_{2}+k_{4}\right|(3.10\right.
\end{aligned}
$$

Expressions (3.9), (3.10) were found in the original papers of the author (V.Zakharov, 1967; V.Zakharov, 1968). Formulae (3.7), (3.8) were published in 1970 (V.Zakharov, Kharitonov, 1970). (See also, A.Radder, 1992; R.Lin, W.Perrie, 1997.)

In the present moment the calculation of $L^{(n)}$ for any $n$ is a relatively easy problem. Kinetic energy can be presented in the form

$$
T=\frac{1}{2} \int \Psi \hat{G} \Psi d r
$$

where $\hat{G}=\hat{G}(\eta)$ is the Dirichet-Neumann Operator, defined as

$$
\hat{G}(\eta) \Psi=\left.\left(1+\nabla_{\perp} \eta\right)^{1 / 2} \frac{\partial \Phi}{\partial \eta}\right|_{z=\eta}
$$

This operator is self-adjoint, non-negative, and can be presented in a form of series in powers of $\eta$ :

$$
G=\sum_{n=0}^{\infty} G_{n}
$$

where

$$
G_{0}=\left|\nabla_{\perp}\right| \tanh \left(\left|\nabla_{\perp}\right| h\right)
$$

The terms $G_{n}$ may be computed systematicaly by the use of a recursion formula found by Craig and Sulem (Craig, Sulem, 1992). (See also, Craig, Groves, 1994.)

The potential energy $U$ can be expanded in Taylor series on $\nabla_{\perp} \eta$ by the use of expansion on $\sqrt{1+\nabla_{\perp} \eta^{2}}$ in (2.5). Finally, we obtain: 


$$
H=H_{0}+H_{1}+H_{2}+\cdots
$$

The first three terms in this expansion are the following:

$$
\begin{aligned}
H_{0}= & \frac{1}{2} \int\left\{A_{k}\left|\Psi_{k}\right|^{2}+B_{k}\left|\eta_{k}\right|^{2}\right\} d k \\
& A_{k}=k \tanh (k h), \quad B_{k}=g+\sigma k^{2} \\
H_{1}= & \frac{1}{2(2 \pi)} \int L^{(1)}\left(k_{1}, k_{2}\right) \Psi_{k_{1}} \Psi_{k_{2}} \eta_{k_{3}} \delta\left(k_{1}+k_{2}+k_{3}\right) d k_{1} d k_{2} d k_{3} \\
H_{2}= & \frac{1}{2(2 \pi)^{2}} \int L^{(2)}\left(k_{1}, k_{2}, k_{3}, k_{4}\right) \Psi_{k_{1}} \Psi_{k_{2}} \eta_{k_{3}} \eta_{k_{4}} \delta\left(k_{1}+k_{2}+k_{3}+k_{4}\right) d k_{1} d k_{2} d k_{3} d k_{4}- \\
& -\frac{\sigma^{2}}{8(2 \pi)^{2}} \int\left(k_{1}, k_{2}\right)\left(k_{3}, k_{4}\right) \eta_{k_{1}} \eta_{k_{2}} \eta_{k_{3}} \eta_{k_{4}} \delta\left(k_{1}+k_{2}+k_{3}+k_{4}\right) d k_{1} d k_{2} d k_{3} d k_{4}
\end{aligned}
$$

\section{Normal Variables}

The Fourier transforms, $\Psi_{k}$ and $\eta_{k}$, satisfy the conditions:

$$
\begin{aligned}
\Psi_{-k} & =\Psi_{k}^{*} \\
\eta_{-k} & =\eta_{k}^{*} .
\end{aligned}
$$

They can be given as:

$$
\begin{aligned}
\eta_{k} & =\frac{1}{\sqrt{2}}\left(\frac{A_{k}}{B_{k}}\right)^{1 / 4}\left(a_{k}+a_{-k}^{*}\right), \\
\Psi_{k} & =\frac{i}{\sqrt{2}}\left(\frac{B_{k}}{A_{k}}\right)^{1 / 4}\left(a_{k}-a_{-k}^{*}\right), \\
a_{k} & =\frac{1}{\sqrt{2}}\left\{\left(\frac{B_{k}}{A_{k}}\right)^{1 / 4} \eta_{k}-i\left(\frac{A_{k}}{B_{k}}\right)^{1 / 4} \Psi_{k}\right\} .
\end{aligned}
$$

The transformation $\Psi_{k}, \eta_{k} \rightarrow a_{k}$ is canonical in the following sense. One can check that

$$
\frac{\partial a_{k}}{\partial t}+i \frac{\partial H}{\partial a_{k}^{*}}=0
$$

where Hamiltonian $H$ can be presented as a sum of two terms

$$
H=H_{0}+H_{\text {int }} .
$$


For the first term we have

$$
H_{0}=\int \omega_{k} a_{k} a_{k}^{*} d k
$$

where $\omega_{k}>0$, and is defined as

$$
\omega_{k}=\sqrt{A_{k} B_{k}}=\sqrt{k \tanh (k h)\left(g+\sigma k^{2}\right)} .
$$

The second term, $H_{\text {int }}$, is presented by the infinite series:

$$
\begin{aligned}
& H_{i n t}=\frac{1}{n ! m !} \sum_{n+m \geq 3} \int V^{n, m}\left(k_{1} \ldots k_{n}, k_{n+1} \ldots k_{n+m}\right) a_{k_{1}}^{*} \ldots a_{k_{n}}^{*} a_{k_{n+1}} \ldots a_{k_{n+m}} \times \\
& \times \delta\left(k_{1}+\cdots+k_{n}-k_{n+1}-\cdots-k_{n+m}\right) d k_{1} \ldots d k_{n+m}
\end{aligned}
$$

In the case under consideration we have

$$
V^{(n, m)}(P, Q)=V^{(m, n)}(Q, P)
$$

where $P=\left(k_{1} \ldots k_{n}\right)$ and $Q=\left(k_{n+1} \ldots k_{n+m}\right)$ are multyindecies.

For more general Hamiltonian systems (in the presense of wind, for instance), the coefficients $V^{n, m}(P, Q)$ are complex, and

$$
V^{(n, m)}(P, Q)=V^{*(m, n)}(Q, P) .
$$

Condition (4.11) garantees that the Hamiltonian $H_{\text {int }}$ is real.

For surface waves the coefficients can be written as:

$$
\begin{gathered}
V^{(1,2)}\left(k, k_{1}, k_{2}\right)=\frac{1}{4 \pi \sqrt{2}}\left\{\left(\frac{A_{k} B_{k_{1}} B_{k_{2}}}{B_{k} A_{k_{1}} A_{k_{2}}}\right)^{1 / 4} L^{(1)}\left(k_{1}, k_{2}\right)-\right. \\
\left.-\left(\frac{B_{k} A_{k_{1}} B_{k_{2}}}{A_{k} B_{k_{1}} A_{k_{2}}}\right)^{1 / 4} L^{(1)}\left(-k, k_{1}\right)-\left(\frac{B_{k} B_{k_{1}} A_{k_{2}}}{A_{k} A_{k_{1}} A_{k_{2}}}\right)^{1 / 4} L^{(1)}\left(-k, k_{2}\right)\right\} \\
V^{(0,3)}\left(k, k_{1}, k_{2}\right)=\frac{1}{4 \pi \sqrt{2}}\left\{\left(\frac{A_{k} B_{k_{1}} B_{k_{2}}}{B_{k} A_{k_{1}} A_{k_{2}}}\right)^{1 / 4} L^{(1)}\left(k_{1}, k_{2}\right)+\right. \\
\left.+\left(\frac{B_{k} A_{k_{1}} B_{k_{2}}}{A_{k} B_{k_{1}} A_{k_{2}}}\right)^{1 / 4} L^{(1)}\left(k, k_{1}\right)+\left(\frac{B_{k} B_{k_{1}} A_{k_{2}}}{A_{k} A_{k_{1}} A_{k_{2}}}\right)^{1 / 4} L^{(1)}\left(k, k_{2}\right)\right\}
\end{gathered}
$$

In this paper we will use only one coefficient of fourth order $V^{(2,2)}(P, Q)$. After a simple calculation we can obtain the following expression for this coefficient: 


$$
\begin{gathered}
V^{(2,2)}\left(k_{1}, k_{2}, k_{3}, k_{4}\right)=\frac{1}{8 \pi^{2}}\left\{\tilde{L}^{(2)}\left(-k_{1},-k_{2}, k_{3}, k_{4}\right)+\tilde{L}^{(2)}\left(k_{3}, k_{4},-k_{1},-k_{2}\right)-\tilde{L}^{(2)}\left(-k_{1}, k_{3},-k_{2}, k_{4}\right)\right. \\
\left.-\quad \tilde{L}^{(2)}\left(-k_{1}, k_{4},-k_{2}, k_{3}\right)-\tilde{L}^{(2)}\left(-k_{2}, k_{3},-k_{1}, k_{4}\right)-\tilde{L}^{(2)}\left(-k_{2}, k_{4},-k_{1}, k_{3}\right)\right\}- \\
-\frac{\sigma^{2}}{64 \pi^{2}}\left\{\left(k_{1}, k_{2}\right)\left(k_{3}, k_{4}\right)+\left(k_{1}, k_{3}\right)\left(k_{2}, k_{4}\right)+\left(k_{1}, k_{4}\right)\left(k_{2}, k_{3}\right)\right\}\left(\frac{A_{k_{1}} A_{k_{2}} A_{k_{3}} A_{k_{4}}}{B_{k_{1}} B_{k_{2}} B_{k_{3}} B_{k_{4}}}\right)^{1 / 4} \\
\tilde{L}^{(2)}\left(k_{1}, k_{2}, k_{3}, k_{4}\right)=\frac{1}{4}\left(\frac{B_{k_{1}} B_{k_{2}} A_{k_{2}} A_{k_{1}}}{A_{k_{1}} A_{k_{2}} B_{k_{3}} B_{k_{4}}}\right)^{1 / 4} L^{(2)}\left(k_{1}, k_{2}, k_{3}, k_{4}\right)
\end{gathered}
$$

We will not discuss the five-wave processes systematically. This makes it possible to use the following approximation for the Hamiltonian:

$$
\begin{aligned}
& H=\int \omega_{k}\left|a_{k}\right|^{2} d k+ \\
& +\frac{1}{2} \int V^{(1,2)}\left(k, k_{1}, k_{2}\right)\left(a_{k} a_{k_{1}}^{*} a_{k_{2}}^{*}+a_{k}^{*} a_{k_{1}} a_{k_{2}}\right) \delta\left(k-k_{1}-k_{2}\right) d k d k_{1} d k_{2}+ \\
& +\frac{1}{6} \int V^{(0,3)}\left(k, k_{1}, k_{2}\right)\left(a_{k} a_{k_{1}} a_{k_{2}}+a_{k}^{*} a_{k_{1}}^{*} a_{k_{2}}^{*}\right) \delta\left(k+k_{1}+k_{2}\right) d k d k_{1} d k_{2}+ \\
& +\frac{1}{4} \int V^{(2,2)}\left(k, k_{1}, k_{2}, k_{3}\right) a_{k}^{*} a_{k_{1}}^{*} a_{k_{2}} a_{k_{3}} \delta\left(k+k_{1}-k_{2}-k_{3}\right) d k d k_{1} d k_{2} d k_{3}
\end{aligned}
$$

\section{Canonical Transformation}

In this chapter we will study only the gravity waves and put $\sigma=0$. Now:

$$
\omega_{k}=g k \tanh (k h) .
$$

The dispersion relation (5.1) is of the "non-decay type" and the equations

$$
\begin{aligned}
\omega_{k} & =\omega_{k_{1}}+\omega_{k_{2}} \\
k & =k_{1}+k_{a}
\end{aligned}
$$

have no real solution. It means that in the limit of small nonlinearity, the cubic terms in the Hamiltonian (4.16) can be excluded by a proper canonical transformation. The transformation

$$
a(k, t) \rightarrow b(k, t)
$$

must transform equation (4.5) into the same equation:

$$
\frac{\partial b_{k}}{\partial t}+i \frac{\delta H}{\delta b_{k}^{*}}=0 .
$$


This requirement imposes the following conditions on the Poisson's brackets between $a_{k}$ and $b_{k}$ :

$$
\begin{aligned}
\left\{a_{k}, a_{k^{\prime}}\right\} & =\int\left\{\frac{\delta a_{k}}{\delta b_{k^{\prime \prime}}} \frac{\delta a_{k^{\prime}}}{\delta b_{k^{\prime \prime}}^{*}}-\frac{\delta a_{k}}{\delta b_{k^{\prime \prime}}^{*}} \frac{\delta a_{k^{\prime}}}{\delta b_{k^{\prime \prime}}}\right\} d k^{\prime \prime}=0 \\
\left\{a_{k}, a_{k^{\prime}}^{*}\right\} & =\int\left\{\frac{\delta a_{k}}{\delta b_{k^{\prime \prime}}} \frac{\delta a_{k^{\prime}}^{*}}{\delta b_{k^{\prime \prime}}^{*}}-\frac{\delta a_{k}}{\delta b_{k^{\prime \prime}}^{*}} \frac{\delta a_{k^{\prime}}^{*}}{\delta b_{k^{\prime \prime}}}\right\} d k^{\prime \prime}=\delta\left(k-k^{\prime}\right) \\
\left\{b_{k}, b_{k^{\prime}}\right\} & =\int\left\{\frac{\delta b_{k}}{\delta a_{k^{\prime \prime}}} \frac{\delta b_{k^{\prime \prime}}}{\delta a_{k^{\prime}}^{*}}-\frac{\delta b_{k}}{\delta a_{k^{\prime \prime}}^{*}} \frac{\delta b_{k^{\prime \prime}}}{\delta a_{k^{\prime}}}\right\} d k^{\prime \prime}=0 \\
\left\{b_{k}, b_{k^{\prime}}^{*}\right\} & =\int\left\{\frac{\delta b_{k}}{\delta a_{k^{\prime \prime}}} \frac{\delta b_{k^{\prime \prime}}^{*}}{\delta a_{k^{\prime}}^{*}}-\frac{\delta b_{k}}{\delta a_{k^{\prime \prime}}^{*}} \frac{\delta b_{k^{\prime \prime}}^{*}}{\delta a_{k^{\prime}}}\right\} d k^{\prime \prime}=\delta\left(k-k^{\prime}\right)
\end{aligned}
$$

The canonical transformation excluding cubic terms is given by the infinite series:

$$
\begin{aligned}
a_{k} & =a_{k}^{(0)}+a_{k}^{(1)}+a_{k}^{(2)}+\cdots \\
a_{k}^{(0)} & =b_{k} \\
a_{k}^{(1)} & =\int \Gamma_{k, k_{1}, k_{2}}^{(1)} b_{k_{1}} b_{k_{2}} \delta\left(k-k_{1}-k_{2}\right) d k_{1} d k_{2}-2 \int \Gamma_{k_{2}, k, k_{2}}^{(1)} b_{k_{1}}^{*} b_{k_{2}} \delta\left(k+k_{1}-k_{2}\right) d k_{1} d k_{2}+ \\
& +\int \Gamma_{k, k_{1}, k_{2}}^{(2)} b_{k_{1}}^{*} b_{k_{2}}^{*} \delta\left(k+k_{1}+k_{2}\right) d k_{1} d k_{2} \\
a_{k}^{(2)} & =\int B_{k, k_{1}, k_{2}, k_{3}} b_{k_{1}}^{*} b_{k_{2}} b_{k_{3}} \delta\left(k+k_{1}-k_{2}-k_{3}\right) d k_{1} d k_{2} d k_{3}+\cdots
\end{aligned}
$$

Plugging (5.9) to (5.5), (5.6), we obtain infinite series in powers of $b, b^{*}$, which have to be identically zero in all orders exept zero.

Let us accept

$$
\Gamma^{(2)}\left(k, k_{1}, k_{2}\right)=\Gamma^{(2)}\left(k_{1}, k, k_{2}\right)=\Gamma^{(2)}\left(k_{2}, k, k_{1}\right) .
$$

This condition provides that (5.5), (5.6) are satisfied in the first order on $b, b^{*}$. Substituting (5.9) to $H$ we observe that cubic terms vanish:

$$
\begin{aligned}
\Gamma^{(1)}\left(k, k_{1}, k_{2}\right) & =-\frac{1}{2} \frac{V^{(1,2)}\left(k, k_{1}, k_{2}\right)}{\left(\omega_{k}-\omega_{k_{1}}-\omega_{k_{2}}\right)} \\
\Gamma^{(2)}\left(k, k_{1}, k_{2}\right) & =-\frac{1}{2} \frac{V^{(0,3)}\left(k, k_{1}, k_{2}\right)}{\left(\omega_{k}+\omega_{k_{1}}+\omega_{k_{2}}\right)}
\end{aligned}
$$

To calculate quadratic terms in the Hamiltonian, one has to calculate $B\left(k, k_{1}, k_{2}, k_{3}\right)$. It can be done by putting zero quadratic in $b, b^{*}$, terms in (5.5), (5.6). This condition imposes on $B\left(k, k_{1}, k_{2}, k_{3}\right)$ the system of linear inhomogenous equations, which can be solved explicitly. This time-consuming work was done by V.Krasitskii (Krasitskii, 1991). 
In this paper we offer another method for the construction of the canonical transformation, making possible to calculate $b_{k}$ on all orders on $b_{k}, b_{k}^{*}$. Let us indtoduce a new variable $\tau$, "auxiliary time", and consider the following Hamiltonian system:

$$
\frac{\partial a_{k}}{\partial \tau}+i \frac{\delta R}{\delta a_{k}^{*}}=0,
$$

where

$$
\begin{aligned}
R & =+i \int \Gamma^{(1)}\left(k, k_{1}, k_{2}\right)\left(a_{k}^{*} a_{k_{1}} a_{k_{2}}-a_{k} a_{k_{1}}^{*} a_{k_{2}}^{*}\right) \delta\left(k-k_{1}-k_{2}\right) d k d k_{1} d k_{2} \\
& +\frac{i}{3} \int \Gamma^{(2)}\left(k, k_{1}, k_{2}\right)\left(a_{k}^{*} a_{k_{1}}^{*} a_{k_{2}}^{*}-a a_{k} a_{k_{1}} a_{k_{2}} \delta\left(k+k_{1}+k_{2}\right) d k d k_{1} d k_{2}\right.
\end{aligned}
$$

and solve the equation (5.13) imposing the "initial condition"

$$
\left.a_{k}\right|_{\tau=0}=b_{k}
$$

The following statement holds:

Solution of the equation (5.13) at $\tau=1$ represents the canonical transformation (5.9).

To prove this fact we mention that in virtue of (5.13) the mapping $a(k, \tau) \rightarrow b(k, \tau)$ is canonical for any $\tau$. The equation (5.13) is:

$$
\begin{aligned}
\frac{\partial a_{k}}{\partial \tau} & =\int \Gamma^{(1)}\left(k, k_{1}, k_{2}\right) a_{k_{1}} a_{k_{2}} \delta\left(k-k_{1} k_{2}\right) d k_{1} d k_{2} \\
& -2 \int \Gamma^{(1)}\left(k_{2}, k, k_{1}\right) a_{k_{1}}^{*} a_{k_{2}} \delta\left(k+k_{1}+k_{2}\right) d k_{1} d k_{2}+ \\
& +\int \Gamma^{(2)}\left(k, k_{1}, k_{2}\right) a_{k_{1}}^{*} a_{k_{2}}^{*} \delta\left(k+k_{1}+k_{2}\right) d k_{1} d k_{2}
\end{aligned}
$$

Expanding $a_{k}$ in Taylor series on $\tau$, one can find that the first term in this expansion coincides with quadratic terms $b^{(1)}$ in (5.9). Hence, we found the desired transformation.

$$
\begin{aligned}
& B\left(k, k_{1}, k_{2}, k_{3}\right)=\Gamma^{(1)}\left(k_{1}, k_{2}, k_{1}-k_{2}\right) \Gamma^{(1)}\left(k_{3}, k, k_{3}-k\right)+ \\
& \Gamma^{(1)}\left(k_{1}, k_{3}, k_{1}-k_{3}\right) \Gamma^{(1)}\left(k_{2}, k, k_{2}-k\right)- \\
- & \Gamma^{(1)}\left(k, k_{2}, k-k_{2}\right) \Gamma^{(1)}\left(k_{3}, k_{1}, k_{3}-k_{1}\right)-\Gamma^{(1)}\left(k_{1}, k_{3}, k_{1}-k_{3}\right) \Gamma^{(1)}\left(k_{2}, k_{1}, k_{2}-k_{1}\right)- \\
- & \left.\Gamma^{(1)}\left(k+k_{1}, k, k_{1}\right) \Gamma^{(1)}\left(k_{2}+k_{3}, k_{2}, k_{3}\right)+\Gamma^{(2)}\left(-k-k_{1}, k, k_{1}\right) \Gamma^{(2)}\left(-k_{2}-k_{3}, k_{2}, k_{5}\right) 17\right)
\end{aligned}
$$

The expression (5.17) was found in 1991 (Zakharov, 1992). It coincided the Krasitskii equation found approximately at the same time but by the use of different methods.

To find all $a^{(1)}$ we can use the representation: 


$$
a(k, \tau)=\sum_{n=0}^{\infty} a^{(n)} \tau^{n},
$$

substitute (5.18) to (5.13) and equalize terms of the power $n$. This procedure gives the recursion relation for calculation of $a^{(n)}$.

\section{Shallow Water Approximation}

The theory of surface waves can be essentially simplified in the "shallow water" limit $k h \rightarrow 0$. This approximation, first introduced by Airy (Airy,1847) can be realized in terms of Hamiltonian formalism by two different ways.

The first way (Craig, Groves, 1994) is the most straightforward. We can present the solution of the Laplace equation

$$
\nabla \Phi=0
$$

in the form

$$
\Phi=\Phi_{0}+\sum_{n=1}^{\infty} \frac{1}{2 n(2 n-1)} \Phi_{n}(z+h)^{2 n},
$$

where $\Phi_{0}, \Phi_{n}$ are functions on $r$, and

$$
\Phi_{n+1}=-\Delta_{\perp} \Phi_{n}=-\left(\frac{\partial^{2}}{\partial x^{2}}+\frac{\partial^{2}}{\partial y^{2}}\right) \Phi_{n} .
$$

The solution (6.3) satisfies the boundary condition on the bottom:

$$
\left.\frac{\partial \Phi}{\partial z}\right|_{z=-h}=0
$$

To satisfy the condition on a free surface we have to put:

$$
\Psi=\Phi_{0}+\sum_{n=1}^{\infty} \frac{(-1)^{n}}{2 n(2 n-1)}\left(\Delta_{\perp}^{n} \Phi_{0}\right)(\eta+h)^{2 n} .
$$

We assume that $\eta \leq h$. The expansion in $(6.5)$ is going in powers of the parameter $(k h)^{2}$. Equation (6.5) can be solved by iteration

$$
\Phi_{0}=\Psi+\Psi^{(1)}+\cdots,
$$

where the second term is:

$$
\Psi^{(1)}=\frac{1}{2} \Delta_{\perp} \Psi
$$

For the kinetic energy we have: 


$$
\begin{aligned}
T & =T^{(0)}+T^{(1)}+\cdots, \\
T^{(0)} & =\frac{1}{2} \int(\eta+h)\left(\Delta_{\perp} \Psi\right)^{2} d r, \\
T^{(1)} & =-\frac{1}{6} \int(\eta+h)^{3}\left(\Delta_{\perp} \Psi\right)^{2} d r-\frac{1}{2} \int(\eta+h)^{2}\left(\nabla_{\perp} \eta \nabla_{\perp} \Psi\right) \Delta_{\perp} \Psi d r .
\end{aligned}
$$

Further we will study the approximation of a shallow water in combination with the approximation of small angles. In this limit $T^{(1)}$ can be simplified to the form

$$
T^{(1)}=-\frac{h^{3}}{6} \int\left(\Delta_{\perp} \Psi\right)^{2} d r .
$$

In this approximation Hamiltonian $H$ is:

$$
\begin{aligned}
H & =H_{0}+H_{1}, \\
H_{0} & =\frac{h}{2} \int\left(\nabla_{\perp} \Psi\right)^{2} d r-\frac{h^{3}}{6} \int\left(\Delta_{\perp} \Psi\right)^{2} d r+\frac{g}{2} \int \eta^{2} d r+ \\
& +\frac{\sigma}{2} \int\left(\nabla_{\perp} \Psi\right)^{2} d r, \\
H_{1} & =\frac{1}{2} \int \eta\left(\nabla_{\perp} \Psi\right)^{2} d r .
\end{aligned}
$$

The motion equations are:

$$
\begin{aligned}
& \frac{\partial \eta}{\partial t}+\operatorname{div}(\eta+h) \nabla_{\perp} \Psi=-\frac{h^{3}}{3} \Delta_{\perp}^{2} \Psi \\
& \frac{\partial \Psi}{\partial t}+\frac{1}{2}\left(\nabla_{\perp} \Psi\right)^{2}+g \eta=\sigma \Delta_{\perp} \eta .
\end{aligned}
$$

In one-dimensional geometry:

$$
\begin{aligned}
\frac{\partial \eta}{\partial t}+\frac{\partial}{\partial x}(\eta+h) \frac{\partial \Psi}{\partial x} & =-\frac{h^{3}}{3} \frac{\partial^{2} \Psi}{\partial x^{2}}, \\
\frac{\partial \Psi}{\partial t}+\frac{1}{2}\left(\frac{\partial \Psi}{\partial x}\right)^{2}+g \eta & =\sigma \frac{\partial^{2} \eta}{\partial x^{2}}
\end{aligned}
$$

System (6.16) is completely integrable if $\sigma=0$ (Kaup, 1976). It makes the whole system (6.14), (6.15) interesting from a pure theoretical point of view. However, this system is ill-posed in the region of very short waves. The linear analysis of (6.14), (6.15) gives the following expression for the dispersion relation: 


$$
\omega_{k}^{2}=k h\left(g+\sigma k^{2}\right)\left(1-\frac{1}{3}(k h)^{2}\right)
$$

if $1 / 3(k h)^{2}>1$. This is far beyond the limits of the applicability of the system (6.14), (6.15). Thus, this "instability" is nonphysical. Howeve, this ill-poseness is the obstacle for the numerical simulation of this system.

\section{Regularized Shallow-Water Equations}

There are many different ways to regularized the ill-posed system (6.14)-(6.15). Usually, the regularization modifies the dispersion relation of surface waves. We offer below one more way for regularization of mentioned system, keeping untouched the dispersion relation:

$$
\omega_{k}=\sqrt{\left(g_{k}+\sigma k^{3}\right) \tanh k} .
$$

We preserve the exact formula for $H_{0}$ and will perform the transition $k h \rightarrow 0$ only in the interaction Hamiltonian $H_{\text {int }}$. This transition leads to a very simple expression

$$
H_{\text {int }}=\frac{1}{2} \int \eta\left(\nabla_{\perp} \Psi\right)^{2} d r
$$

To justify this choice, one can compare $T^{(1)}$ given by (6.10), and $T^{(0)}$ given by (6.9). Since $\eta$ can be at most of the order of $h$, wee can conclude that:

$$
T^{(1)} \simeq(k h)^{2} T^{(0)}
$$

and more generally

$$
T^{(n)} \simeq(k h)^{2 n} T^{(0)} .
$$

High-order terms in potential energy are very small as well.

Finally, we obtain for the Hamiltonian:

$$
\begin{aligned}
H & =\frac{1}{2} \int\left[k \tanh (k h)\left|\Psi_{k}\right|^{2}+\left(g+\sigma k^{2}\right)\left|\eta_{k}\right|^{2}\right] d k- \\
& -\frac{1}{4 \pi} \int\left(k_{1} k_{2}\right) \Psi_{k_{1}} \Psi_{k_{2}} \eta_{k_{3}} \delta\left(k_{1}+k_{2}+k_{3}\right) d k_{1} d k_{2} d k_{3}
\end{aligned}
$$

The motion equations become:

$$
\begin{aligned}
\frac{\partial \eta}{\partial t} & =k \tanh (k h) \Psi_{k}-\frac{1}{2 \pi} \int\left(k k_{1}\right) \Psi_{k_{1}} \eta_{k_{2}} \delta\left(k-k_{1}-k_{2}\right) d k_{1} d k_{2} \\
\frac{\partial \Psi}{\partial t} & =-\left(g+\sigma k^{2}\right) \eta_{k}+\frac{1}{4 \pi} \int\left(k_{1} k_{2}\right) \Psi_{k_{1}} \Psi_{k_{2}} \delta\left(k-k_{1}-k_{2}\right) d k_{1} d k_{2}
\end{aligned}
$$




$$
\begin{aligned}
& \frac{\partial \eta}{\partial t}+\operatorname{div} \eta \nabla \Psi=\hat{L} \Psi=\left|\nabla_{\perp}\right| \tanh \left|\nabla_{\perp}\right| h \Psi \\
& \frac{\partial \Psi}{\partial t}+\frac{1}{2}(\nabla \Psi)^{2}+g \eta=\sigma \nabla \eta .
\end{aligned}
$$

Equations (7.6)-(7.9) are very convinient for the direct numerical simulation by the use of a spectral code. In (7.8) $\hat{L}$ is a pseudodifferential operator of convolution type, $(\hat{L} \Psi)_{k}=k \tanh (k h) \Psi_{k}$. It would be very interesting to know how good solitons in the system (7.8)-(7.9) describe real solitons.

\section{Kadomtsev - Petviashvili Equation}

Another way for combining both basic approximations is to perform the limit $k h \rightarrow 0$ in equations (4.5) after introducing normal variables. We can perform the expansion:

$$
\begin{aligned}
\omega_{k} & =s k\left(1-\alpha k^{2}+\cdots\right), \\
\alpha & =\frac{1}{2}\left(\frac{1}{3} h^{2}-\frac{\sigma}{g}\right),
\end{aligned}
$$

where coefficient $\alpha$ defines the dispersion of waves on shallow water. For deeper water:

$$
h>h_{0} \simeq \sqrt{\frac{3 \sigma}{g}} \sim 3 \mathrm{~cm},
$$

$\alpha>0$, and the dispersion is negative. We will discuss this case further. For $h<h_{0}$ we have $\alpha<0$, and the dispersion is positive.

Calculating $H_{\text {int }}$, we can put:

$$
A_{k} \simeq h k^{2}, \quad B_{k} \simeq g
$$

Then:

$$
\begin{gathered}
V^{(1,2)}\left(k, k_{1}, k_{2}\right)=V^{(0,3)}\left(k, k_{1}, k_{2}\right)=V\left(k, k_{1}, k_{2}\right)= \\
=\frac{1}{4 \pi \sqrt{2}}\left(\frac{g}{h}\right)^{1 / 4}\left(k k_{1} k_{2}\right)^{1 / 2}\left\{\frac{\left(k k_{1}\right)}{k k_{1}}+\frac{\left(k k_{2}\right)}{k k_{2}}+\frac{\left(k_{1} k_{2}\right)}{k_{1} k_{2}}\right\}
\end{gathered}
$$

This expression behaves at $k h \rightarrow 0$ like $(k h)^{-1 / 4}$. Analysis of expressions (3.8), (4.14) show that:

$$
V^{(2,2)}\left(k, k_{1}, k_{2}, k_{3}\right) \simeq(k h) k^{3}, \quad k h \rightarrow 0
$$


The similar statement holds for all high-order coefficients. One can check that

$$
V^{(n, m)}(P, Q) \rightarrow 0 \quad \text { at } \quad k h \rightarrow 0
$$

for $n+m>3$. Indeed, if $(n+m)$ is odd, then:

$$
T^{n+m-2} \sim \eta^{n+m-2} \Psi^{2} \sim h^{(n+m-2) / 4} .
$$

If $(n+m)$ is even,

$$
T^{n+m-2} \simeq h \eta^{n+m-2} \Psi^{2} \simeq h^{(n+m-2) / 4+1} .
$$

Thus one can neglect all interaction terms except cubical.

Further simplification can be done if we suppose that all excited waves propogate almost in the same direction, suppose along the axis $x$. We denote in this case: $k_{x}=p, k_{y}=q$, $q \ll p$, and $a(q, p)=0$, if $p<0$. In this approximation we have to put:

$$
\begin{aligned}
& \omega(k)=\omega(p, q)=s\left(p+\frac{1}{2} \frac{q^{2}}{p}-\alpha p^{3}\right) \\
& V\left(k, k_{1}, k_{2}\right)=\frac{3}{4 \pi \sqrt{2}}\left(\frac{g}{h}\right)^{1 / 4}\left(p p_{1} p_{2}\right)^{1 / 2} \theta(p) \theta\left(p_{1}\right) \theta\left(p_{2}\right), \\
& \eta_{k}=\frac{1}{\sqrt{2}}\left(\frac{h}{g}\right)^{1 / 4}|p|^{1 / 2}\left(a_{p, q}+a_{-p,-q}^{*}\right) .
\end{aligned}
$$

Here $\theta(p)=1$ if $p>0$, and $\theta(p)=0$ if $p<0$.

The equation (4.5) can be rewritten in the form:

$$
\begin{aligned}
& \frac{\partial a_{p q}}{\partial t}+i \frac{\delta H}{\delta a_{p, q}^{*}}=0 \\
& \frac{\partial a_{p q}}{\partial t}+i\left(s p+\frac{1}{2} \frac{q^{2}}{p}-\alpha p^{3}\right) a_{p q}+i \frac{3}{8 \pi \sqrt{2}}\left(\frac{g}{h}\right)^{1 / 4} \int_{p_{i}>0}\left(p p_{1} p_{2}\right)^{1 / 2} \times \\
& \times\left\{a_{p_{1} q} a_{p_{2}, q} \delta\left(p-p_{1}-p_{2}\right) \delta\left(q-q_{1}-q_{2}\right)+\right. \\
& \left.+2 a_{p_{1}, q_{1}} a_{p_{2}, q_{2}}^{*} \delta\left(p-p_{1}+p_{2}\right) \delta\left(q+q_{1}+q_{2}\right)\right\} d p_{1} d p_{2} d q_{1} d q_{2}
\end{aligned}
$$

By use of (8.10) we can transform (8.12) to the equation on $\eta(p, q)$ :

$$
\begin{aligned}
& \frac{\partial \eta_{p q}}{\partial t}+i s\left(p+\frac{1}{2} \frac{q^{2}}{p}-\alpha p^{3}\right) \eta_{p q}+ \\
& \frac{3}{8 \pi} i p \frac{s}{h} \int \eta_{p_{1} q_{1}} \eta_{p_{2} q_{2}} \delta\left(p-p_{1}-p_{2}\right) \delta\left(q-q_{1}-q_{2}\right) d p_{1} d p_{2} d q_{1} d q_{2}=0
\end{aligned}
$$


Performing the Inverse Fourier transform we obtain:

$$
\frac{\partial \eta}{\partial t}+s \frac{\partial}{\partial x}\left\{\eta+\frac{3}{4} \frac{\eta^{2}}{h}+\frac{1}{2} \int_{\infty}^{x} d x \frac{\partial^{2} \eta}{\partial y^{2}}+\alpha \frac{\partial^{3} \eta}{\partial x^{3}}\right\}=0
$$

This is called the so-called Kadomtsev - Petviashvili (KP) equation. There are two types of KP-equations in dependence on the sign of $\alpha$. For deep water $\alpha>1$, in this case (8.14) is KP-2 equation. For shallow water $\alpha<0$, and (8.14) is KP-1 equation.

The dispersion relation (8.8) has a singularity at $p=0$. To eliminate it, we will put

$$
a(0, q)=0 \quad \text { or } \quad \int \eta d x=0 .
$$

This condition is compatible with the equation (8.11) and holds in time. By the change of variables:

$$
\begin{aligned}
& \frac{\partial}{\partial t}+s \frac{\partial}{\partial x} \rightarrow \frac{\partial}{\partial \tau} \\
& \eta=8 u \\
& \frac{\partial}{\partial x} \rightarrow \frac{1}{|\alpha|^{1 / 4}} \frac{\partial}{\partial x} \\
& \frac{\partial}{\partial y} \rightarrow \frac{\sqrt{6}}{|\alpha|^{1 / 4}} \frac{\partial}{\partial y}
\end{aligned}
$$

the equation(8.14) can be transformed to a "standart" KP equation:

$$
\frac{\partial}{\partial x}\left(\frac{\partial u}{\partial t}+6 u \frac{\partial u}{\partial x}+\frac{\partial^{3} u}{\partial x^{3}}\right)+3 \beta^{2} \frac{\partial^{2} u}{\partial y^{2}}=0
$$

where $\beta^{2}= \pm 1$. For the case of negative dispersion, $\alpha>0, \beta^{2}=1$, equation (8.17) is the standart form for $K P-2$ equation. In the case of positive dispersion, $\alpha<0, \beta^{2}=-1$, equation (8.17) is the $K P-1$ equation written in the standart notation.

Introducing normal variables:

$$
u(p, q)=\frac{1}{\sqrt{2}}\left(a_{p, q}+a_{-p,-q}^{*}\right)
$$

we can realize that $a_{p, q}$ satisfies the equation (8.11), and obtain the following expression for the Hamiltonian:

$$
\begin{aligned}
H= & H_{0}+H_{\text {int }}, \\
H_{0}= & \int_{p>0} \omega(p, q)\left|a_{p q}\right|^{2} d p d q, \quad\left(\omega(p, q)=p^{3}-\frac{3 \beta^{2} q^{2}}{p}\right) \\
H_{\text {int }}= & \frac{1}{4 \pi \sqrt{2}} \int_{p_{i}>0}\left(p_{1} p_{2} p_{3}\right)^{1 / 2}\left\{a_{p_{1} q_{1}}^{*} a_{p_{2} q_{2}} a_{p_{3} q_{3}}+a_{p_{1} q_{1}} a_{p_{1} q_{2}}^{*} a_{p_{3} q_{3}}^{*}\right\} \times \\
& \times \delta\left(p-p_{1}-p_{2}\right) \delta\left(q-q_{1}-q_{2}\right) d p d p_{1} d p_{2} d q d q_{1} d q_{2} .
\end{aligned}
$$


Let us consider the equation (8.17) in the rectangular domain $0<x<L_{1}, 0<y<L_{2}$, $S=L_{1} L_{2}$. Now $p, q$ run a discrete set of values

$$
\begin{aligned}
& p_{n}=\frac{2 \pi}{L_{1}} n, \\
& q_{n}=\frac{2 \pi}{L_{2}} m,
\end{aligned}
$$

where $1 \leq n<\infty$ are positive integers, and $-\infty<m<\infty$ are all integers including zero.

Assuming that

$$
u=\frac{1}{\sqrt{2 s}} \sum_{n, m}\left|p_{n}\right|^{1 / 2}\left\{a_{n m} e^{i\left(p_{n} x+q_{m} y\right)}+a_{n m}^{*} e^{-i\left(p_{n} x+q_{m} y\right)}\right\},
$$

we can check that $a_{n m}$ satisfies the Hamiltonian system:

$$
\begin{gathered}
\frac{\partial a_{n m}}{\partial t}+i \frac{\partial \hat{H}}{\partial a_{n m}^{*}}=0 \\
\hat{H}=\sum_{n, m} \omega\left(p_{n} q_{m}\right)\left|a_{n m}\right|^{2}+\frac{1}{2 \sqrt{2 S}} \sum_{n_{i}, m_{i}>0}\left(p_{n_{1}} p_{n_{2}} p_{n_{3}}\right)^{1 / 2} \times \\
\quad \times \delta\left(n-n_{1}-n_{2}\right) \delta\left(m-m_{1}-m_{2}\right)\left[a_{n_{1} m_{1}}^{*} a_{n_{2} m_{2}} a_{n_{3} m_{3}}+a_{n_{1} m_{1}}^{*} a_{n_{2} m_{2}}^{*} a_{n_{3} m_{3}}^{*}\right] .
\end{gathered}
$$

Here Hamiltonian $\hat{H}$ is the energy of fluid inside the rectangular $0<x<L_{1}, 0<y<L_{2}$.

One can define the Poisson brackets between two complex functions $A\left(a_{n m}, a_{n m}^{*}\right)$, $B\left(a_{n m}, a_{n m}^{*}\right)$ as follow:

$$
\{A, B\}=\sum_{n, m>0}\left(\frac{\partial A}{\partial a_{n m}} \frac{\partial B}{\partial a_{n m}^{*}}-\frac{\partial B}{\partial a_{n m}^{*}} \frac{\partial A}{\partial a_{n m}}\right) .
$$

In $(8.22)$

$$
\omega\left(p_{n}, q_{m}\right)=p_{n}^{3}-\frac{3 \beta^{2}}{p_{n}} q_{m}^{2}
$$

The KP equations have an infinite number of conservative quantities:

$$
\begin{aligned}
& \frac{d I_{n}}{d t}=0, \\
& I_{0}=\int u d x d y, \quad\left(w_{x}=\beta u_{y}\right) \\
& I_{1}=\int w d x d y, \quad \\
& I_{2}=\int u^{2} d x d y
\end{aligned}
$$




$$
\begin{aligned}
I_{3} & =\int u w d x d y \\
I_{4} & =\int\left(\frac{1}{2} u_{x}^{2}-\frac{3}{2} \beta^{2} w^{2}+u^{3}\right) d x d y, \\
I_{5} & =\int\left\{w u_{y}-u_{x} w_{x}+2 w u^{2}+\beta w \frac{\partial}{\partial y} \partial^{-1} w\right\} d x d y, \\
I_{6} & =\ldots
\end{aligned}
$$

\section{Integrability of KP-2 Equation}

The KP equation (8.17) can be presented as a compatibility condition for the following linear system imposed on the auxiliary complex function $\Psi$ :

$$
\begin{aligned}
& \left(\beta \frac{\partial}{\partial y}+\frac{\partial^{2}}{\partial x^{2}}+u\right) \Psi=0 \\
& \left(\frac{\partial}{\partial t}+a \frac{\partial^{3}}{\partial x^{3}}+6 u \frac{\partial}{\partial x}+3 u_{x}-3 \beta w\right) \Psi=0
\end{aligned}
$$

where $w_{x}=u_{y}$.

This fact discovered in 1974 (Zakharov, Shabat, 1974; Dryuma, 1974) turned the KP equation to one of the most basic objects of modern nonlinear mathematical physics. An enormous number of articles are devoted to this equation. We can refere the reader to the book of Konopelchenko (Konopelchenko, 1993) and to review articles (Zakharov, Shulman, 1990; Zakharov, Balk, Shulman, 1993). We present below some consequences of representation (9.1), (9.2) which are most important from view-point of the theory of surface waves.

First we have to note that KP-1 and KP-2 are quite different Hamiltonian systems. This difference is very conspicious if we introduce so-called "classical scattering matrix". For any Hamiltonian system (4.5) one can replace the Hamiltonian (4.6) to the auxiliary Hamiltonian

$$
H_{\lambda}=H_{0}+e^{-\lambda|t|} H_{\text {int }}
$$

and tend in (8.11) $t \rightarrow \pm \infty$. For any initial condition

$$
\left.a\right|_{t=0}=a_{0}(k)
$$

we can find

$$
a \rightarrow c_{\lambda}^{ \pm}(k) e^{-i \omega(k) t} \quad t \rightarrow \pm \infty .
$$

The "asymptotic fields" $c_{\lambda}^{ \pm}(k)$ are not independent. They are connected by the relation 


$$
c_{\lambda}^{+}=\hat{S}_{\lambda}\left(c_{\lambda}^{-}\right) .
$$

The operator $\hat{S}_{\lambda}$ is a nonlinear operator presented by the series

$c_{\lambda}^{+s}(k)=c_{\lambda}^{-s}(k)+\sum_{n=2}^{\infty} \sum_{s_{1}, \ldots, s_{n}} \int S_{0,1 \ldots n}(\lambda) c_{1}^{-s_{1}} \cdots c_{n}^{-s_{n}} \delta\left(-s k+s_{1} k_{1}+\cdots+s_{n} k_{n}\right) d k_{1} \ldots d k_{n}$

where $s= \pm 1, c_{k}^{1}=c_{k}, c_{k}^{-1}=c_{k}^{*}$, and

$$
S_{-0,1, \ldots, n}(\lambda)=S_{\lambda}^{-s, s_{1} \ldots, s_{n}}\left(k, k_{1}, \ldots, k_{n}\right)
$$

Series (9.5) converged at least for large enough $\lambda$.

The classical scattering matrix $S$ is defined by the limiting transition

$$
\hat{S}=\lim _{\lambda \rightarrow 0} \hat{S}_{\lambda}, \quad c_{\lambda}^{ \pm}(k) \rightarrow c^{ \pm}(k) .
$$

After this transition the series (9.5) can diverge and turn to the formal series. Anyway, all its terms can be calculated algorithmically. We can easily find that

$$
\begin{aligned}
\lim _{\lambda \rightarrow 0} S_{-0,1, \ldots n}^{(\lambda)}= & 2 \pi i T^{-s, s_{1}, \ldots s_{n}}\left(k k_{1} \ldots k_{n}\right) \times \\
& \times \delta\left(-s \omega_{k}+s_{1} \omega_{1}\left(k_{1}\right)+\cdots+s_{n} \omega_{n}\left(k_{n}\right)\right) .
\end{aligned}
$$

The identity (9.7) is a result of energy conservation.

The coefficients $T^{-s, s_{1} \ldots s_{n}}\left(k, k_{1}, \ldots, k_{n}\right)$ are defined on the resonant manifolds

$$
\begin{aligned}
s k & =s_{1} k_{1}+\cdots+s_{n} k_{n} \\
s \omega_{k} & =s_{1} \omega_{k_{1}}+\cdots+s_{n} \omega_{k_{n}} .
\end{aligned}
$$

A given manifold can occured to exist in dependence on the form of $\omega(k)$. For KP-1 equation we have

$$
\omega(p, q)=p^{3}+\frac{3 q^{2}}{p},
$$

and the first nontrivial manifolds are given by systems:

$$
\begin{array}{lcc}
p=p_{1}+p_{2} & q=q_{1}+q_{2} & \omega(p, q)=\omega\left(p_{1} q_{1}\right)+\omega\left(p_{2} q_{2}\right) \\
p=-p_{1}+p_{2} & q=-q_{1}+q_{2} & \omega(p, q)=-\omega\left(p_{1} q_{1}\right)+\omega\left(p_{2} q_{2}\right) \\
p=p_{1}-p_{2} & q=q_{1}-q_{2} \quad \omega(p, q)=\omega\left(p_{1} q_{1}\right)-\omega\left(p_{2} q_{2}\right)
\end{array}
$$


In this case

$$
\begin{aligned}
T^{(-1,1,1)}\left(k, k_{1}, k_{2}\right) & =T^{(-1,-1,1)}\left(k, k_{1}, k_{2}\right)=T^{(-1,1,-1)}\left(k, k_{1}, k_{2}\right)= \\
=\frac{1}{2} V^{(1,2)}\left(k, k_{1}, k_{2}\right) & =\frac{1}{4 \pi \sqrt{2}}\left(p p_{1} p_{2}\right)^{1 / 2} \theta(p) \theta\left(p_{1}\right) \theta\left(p_{2}\right) .
\end{aligned}
$$

For KP-2 system:

$$
\omega(p, q)=p^{3}-\frac{3 q^{2}}{p},
$$

and equations (9.11) have no real solutions.

The first nontrivial resonant manifold is given by equations:

$$
\begin{aligned}
& p+p_{1}=p_{2}+p_{3} \\
& q+q_{1}=q_{2}+q_{3} \\
& \omega(p, q)+\omega\left(p_{1}, q_{1}\right)=\omega\left(p_{2}, q_{2}\right)+\omega\left(p_{3}, q_{3}\right)
\end{aligned}
$$

The corresponding coefficient in the scattering operator is:

$$
\begin{aligned}
& T^{(-1,-1,1,1)}\left(k, k_{1}, k_{2}, k_{3}\right)=-\frac{1}{2}\left\{\frac{V\left(k+k_{1}, k, k_{1}\right) V\left(k_{2}+K_{3}, k_{2}, k_{3}\right)}{\omega_{k+k_{1}}-\omega_{k}-\omega_{k_{1}}}+\right. \\
& +\frac{V\left(k, k_{2}, k-k_{2}\right) V\left(k_{3}, k_{3}-k_{1}, k_{1}\right)}{\omega_{k-k_{2}}-\omega_{k}+\omega_{k_{3}}}+\frac{V\left(k, k_{3}, k-k_{3}\right) V\left(k_{2}, k_{2}-k_{1}, k_{1}\right)}{\omega_{k-k_{3}}-\omega_{k}+\omega_{k_{3}}}+ \\
& \left.\frac{V\left(k_{2}, k, k_{2}-k\right) V\left(k_{1}, k_{1}-k_{3}, k_{3}\right)}{\omega_{k_{2}-k}+\omega_{k}-\omega_{k_{2}}}+\frac{V\left(k_{3}, k, k_{3}-k\right) V\left(k_{2}, k_{2}-k_{1}, k_{1}\right)}{\omega_{k_{3}-k}+\omega_{k}-\omega_{k_{3}}}\right\}
\end{aligned}
$$

where $V\left(k, k_{1}, k_{2}\right)=V^{(1,2)}\left(k, k_{1}, k_{2}\right)=1 / 2 \pi \sqrt{2}\left(p p_{1} p_{2}\right)^{1 / 2} \theta(p) \theta\left(p_{1}\right) \theta\left(p_{2}\right)$.

The following astonishing statement is of a serious importance for the theory of surface waves:

Theorem 9.1

$$
T^{(-1,-1,1,1)}\left(k, k_{1}, k_{2}, k_{3}\right) \equiv 0
$$

on the resonant manifold (9.14).

This theorem was formulated in 1980 (Zakharov and Shulman, 1980). One can check the identity (9.15) by direct calculations. It is possible, but the calculations are cumbersome and not necessary. Theorem (9.1) is just a very simple consequence of two much stronger theorems:

Theorem 9.2 
For KP-2 equation all the coefficients

$$
T^{-s, s_{1}, \ldots, s_{n}}\left(k, k_{1}, \ldots, k_{n}\right) \equiv 0
$$

on the resonant manifolds (9.8) and the classical scattering matrix is the unit operator.

This theorem was formulated by Zakharov and Shulman in 1988 and corroborates with another theorem, which can be extracted from the work of Bullough, Manakov and Jiang (1988):

Theorem 9.3

Let us consider the Cauchy problem for KP-2 equation:

$$
\left.u\right|_{t=0}=u_{0}(x, y), \quad \int u_{0}(x, y) d x=0 .
$$

If $u_{0}$ is a smooth function and $L_{2}$ norm of $u_{0}$ is small enough:

$$
\left\|u_{0}\right\|^{2}=\int u_{0}^{2}(x, y) d x d y<\epsilon
$$

then:

1. The solution of KP-2 equation exists globally at $-\infty<t<\infty$.

2. Asymptotically at $t \rightarrow \pm \infty$, the function $u(x, y, t)$ tends to be a solution of the linearized equation:

$$
u(x, y, t) \rightarrow \frac{1}{2 \pi} \int c^{ \pm}(p, q) e^{i(p x+q y-\omega(p, q) t)} d p d q .
$$

3. Asymptotic states coincide:

$$
c^{+}(p, q) \equiv c^{-}(p, q)
$$

Theorems (9.2) and (9.3) assert almost identical statements. They were proved almost in the same time, but the methods of proof were cardinally different. To prove (9.3) the authors used the Inverse Scattering Method. This proof is very simple and elegant. It can be generalized and applied to a certain class of systems having a "Lax representation" similar to (9.1), (9.2).

Both KP equations, KP-1 and KP-2, belong to a special class of nonlinear partical differential equations which can be treated by the use of "Inverse Scattering Method". Colloquially, they are called "integrable" equations. This is not exactly correct. The concept of integrability of a finite dimensional Hamiltonian system has the clear mathematical meaning and assumes a possibility of the complete separation of variables in the Hamiltonian - Jacobi equation aand introducing of action-angle variables. This concept can be 
extended to the system of infinite but countable number of degrees of freedom. Nonlinear Hamiltonian PDE in a rectangular domain with periodically boundary conditions are classical examples of such systems.

We (Shulman and myself) have to admit that our proof of the Theorem (9.2) is more difficult and complicated, but it has it's own merits. It is quite elementary proof, which can be extended to a very wide class of nonlinear wave systems.

Let us consider a nonlinear wave Hamiltonian system (4.5),(4.6),(4.7), (4.9) and assume that the wave vector $k$ is 2 -dimensional. We call the dispersion law $\omega_{k}$ degenerative if it safisfies two conditions:

1. The system of equations

$$
\omega_{k+k_{1}}=\omega_{k}+\omega_{k_{1}}
$$

defines in four-dimensional space $\left(k, k_{1}\right)$ a three-dimensional surface $\Gamma$.

2. Another function

$$
f \neq \alpha \omega+\beta k
$$

satisfying on this surface the same equation

$$
f_{k+k_{1}}=f_{k}+\left.f_{k_{1}}\right|_{\Gamma}
$$

can be found. Otherwise the dispersion law is nongenerative.

We can generalize Theorem (9.2) to a following, much more general theorem:

Theorem 9.4

Let us consider the Hamiltonian wave system

$$
\begin{aligned}
& \frac{\partial a_{k}}{\partial t}+i \frac{\delta H}{\delta a_{k}^{*}}=0 \\
& H=\int \omega_{k}\left|a_{k}\right|^{2} d k+\frac{1}{n ! m !} \sum_{n+m \geq 3} \int V^{n, m}\left(k_{1}, \ldots, k_{n}, k_{n+1}, \ldots, k_{n+m}\right) \times \\
& a_{k_{1}}^{*} \ldots a_{k_{n}}^{*} a_{k_{n+1}} \ldots, a_{k_{n+m}} \delta\left(k_{1}+\cdots+k_{n}-k_{n+1}-\ldots-k_{n+m}\right) d k_{1} \ldots d k_{n+m}(9.23)
\end{aligned}
$$

and suppose that

1. The dispersion law $\omega_{k}$ is non-degenerative,

2. System (9.22) has one additional constant of motion I, that is

$$
\frac{d I}{d t}=0
$$

and in virtue of (9.22), (9.23): 


$$
\begin{aligned}
& I_{k}=\int f_{k}\left|a_{k}\right|^{2} d k+\frac{1}{n ! m !} \sum_{n+m \geq 3} \int F^{n, m}\left(k_{1}, \ldots, k_{n}, k_{n+1}, \ldots, k_{n+m}\right) \times \\
& \times a_{k_{1}}^{*} \ldots a_{k_{n}}^{*} a_{k_{n+1}} \ldots a_{k_{n+m}} \delta\left(k_{1}+\cdots+k_{n}+k_{n+1}+\cdots+k_{n+m}\right) d k_{1} \ldots d k_{n+n}(9.2
\end{aligned}
$$

3. All $F^{n, m}\left(k_{1}, \ldots, k_{n}, k_{n+1}, \ldots, k_{n+m}\right)$ are continuous functions of their arguments.

Then:

1. Scattering is trivial. All elements of the scattering operators are identicaly zero on their resonant manifolds,

$$
T^{-s, s_{1}, \ldots, s_{n}}\left(k, k_{1}, \ldots, k_{n}\right) \equiv 0 .
$$

2. System (9.22) has an infinite number of constants of motion similar to (9.24). We can replace $f_{k}$ to any continuous function of two variables $g(k)$ and construct the integral $\tilde{I}$ :

$$
\begin{aligned}
\tilde{I} & =\int g_{k}\left|a_{k}\right|^{2} d k+\cdots \\
\frac{d I}{d t} & =0
\end{aligned}
$$

with all higher-order coefficients $G^{n, m}\left(k_{1} \ldots k_{n+m}\right)$ automatically be continuons.

3. All the constructed integrals commute, the Poisson's brackets between them are zero.

The KP-2 equation "almost satisfies" the conditions of Theorem 9.4. Equation (9.20) has no real solutions if

$$
\omega(p, q)=p^{3}-\frac{3 q^{2}}{p},
$$

so the dispersion law is non-degenerative. Integral $I_{5}$ in (8.26) is the desired additional integral. It's coefficients are not continuous. They have singularities at $p_{i}=0$. However, this flaw can be fixed by imposing the condition $a(0, q)=0$.

It is interesting that KP-1 equation does not satisfy the conditions of Theorem 9.4. The dispersion law

$$
\omega(p, q)=p^{3}+\frac{3 q^{2}}{p}
$$

is degenerative. As a result, scattering in the framework of this equation is nontrivial, and it has much less integrals of motion than KP-2 equation. See on this subject (Zakharov, Shulman; Zakharov, Balk, Shulman).

Practically, Theorem 9.4 claims that system (9.22) is completely integrable. This statement can be made more rigorous if on $(x, y)$ plane system $(9.22)$ is defined inside a rectangular with periodically boundary condition. In a $k$-space we have: 


$$
a_{k}=\sum_{\beta>0} a_{n m} \delta(p-\alpha n) \delta(q-\beta m)
$$

where $\alpha>0$ and $\beta>0$ are some constants.

System (9.22) turns now to a system with a countable number of degrees of freedom:

$$
\dot{a}_{n m}+i \frac{\delta \tilde{H}}{\delta a_{n m}^{*}}=0 .
$$

E.Shulman proved in 1988 the following important theorem (see also Krichever, 1989):

\section{Theorem 9.5}

If the conditions of Theorem 9.4 are satisfied, system (9.28) is integrable in a formal sense.

One can find the canonical transformation

$$
a_{N} \rightarrow c_{n}, \quad N=(n, m), \quad K=(\alpha n, \beta m)
$$

presented by a formal series

$$
a_{N}^{s}=c_{N}^{s}+\sum_{s_{i}, N_{i}} \Gamma^{-, s_{1}, \ldots, s_{n}}\left(k_{1}, k_{2}, \ldots, k_{n}\right) c_{k_{1}}^{s_{1}} \ldots c_{k_{n}}^{s_{n}} \delta\left(s k-s_{1} k_{1}-\cdots-s_{n} k_{n}\right)
$$

such, that the Hamiltonian $\tilde{H}$ has a form:

$$
\tilde{H}=\sum \omega(k)\left|c_{k}\right|^{2}+\sum_{k_{1} \ldots k_{n}} R\left(k_{1}, k_{2}, \ldots, k_{n}\right)\left|c_{k_{1}}\right|^{2} \ldots\left|c_{k_{n}}\right|^{2} .
$$

System (9.28) is integrable in a rigorous sense if series (9.29) and (9.30) are convergent.

System (8.20) satisfies to all formulated conditions and can be transformed to the form (9.30). The canonical transformation transformation (9.29) coincides in quadratic term with the canonical transformation (5.9), excluding cubic terms in the Hamiltonian. In the next order these canonical transformations are different. The final expressions are complicated enough even for the first nontrivial term $R\left(k_{1}, k_{2}\right)$.

\section{One Consequence of Integrability}

A mathematically rigorous proof of integrability of the system (8.22), the KP-2 equation under periodically boundary conditions, would be a big achievment of a pure mathematics. However, even establishing of "formal" integrability of this system, described in the previous chapter, is a serious success. It practice it means the fulfilling of infinite number of very nontrivial identities. We discuss below the simpliest among these identities - 
one particular case of identity (8.25). Intriducing the standart notation $k_{i}=\left(p_{i}, q_{i}\right)$, we assume:

$$
q=0 \quad p_{2}<q \quad p_{3}<p
$$

Under these assumptions the identity

$$
T^{-1,-1,1,1}\left(k, k_{1}, k_{2}, k_{3}\right)=0
$$

on the manifold

$$
\begin{aligned}
& k+k_{1}=k_{2}+k_{3} \\
& \omega_{k}+\omega_{k_{1}}=\omega_{k_{2}}+\omega_{k_{3}}
\end{aligned}
$$

is equivalent to the identity

$$
\begin{aligned}
& -\frac{1}{16 \pi^{2}}\left(p p_{1} p_{2} p_{3}\right)^{1 / 2}\left\{\frac{\left(p+p_{1}\right)}{\left(p+p_{1}\right)^{3}-p^{3}-p_{1}^{3}-\frac{3 q_{1}^{2}}{p+p_{1}}+\frac{3 q_{1}^{2}}{p_{1}}}+\right. \\
& \left.+\frac{\left(p-p_{2}\right)}{\left(p-p_{2}\right)^{3}-p^{3}+p_{2}^{3}-\frac{3 q_{2}^{2}}{p-p_{2}}+\frac{3 q_{2}^{2}}{p_{2}}}+\frac{\left(p-p_{3}\right)}{\left(p-p_{3}\right)^{2}-p^{3}+p_{3}^{3}-\frac{3 q_{3}^{2}}{p-p_{3}}+\frac{3 q_{3}^{2}}{p_{3}}}\right\}=0
\end{aligned}
$$

if

$$
\begin{aligned}
& p+p_{1}=p_{3}+p_{4} \\
& q_{1}=q_{2}+q_{3} \\
& p^{3}+p_{1}^{3}-\frac{3 q_{1}^{2}}{p_{1}}=p_{2}^{3}+p_{3}^{3}-\frac{3 q_{2}^{2}}{p_{2}}-\frac{3 q_{3}^{2}}{p_{3}}
\end{aligned}
$$

The identity (10.5) can be transformed to the form:

$$
\frac{p_{1}\left(p+p_{1}\right)^{2}}{p_{1}^{2}\left(p+p_{1}\right)^{2}+q_{1}^{2}}=\frac{p_{2}\left(p-p_{2}\right)^{2}}{p_{2}^{2}\left(p-p_{2}\right)^{2}+q_{2}^{2}}+\frac{p_{3}\left(p-p_{3}\right)^{2}}{p_{3}^{2}\left(p-p_{3}\right)^{2}+q_{3}^{2}}
$$

It is held on the manifold (10.6)-(10.8). A direct ckecking of this fact is a difficult task. Let us do this in a simple case $p_{3}=p$. Than $p_{1}=p_{2}$, and from $(10.7),(10.8)$ we can obtain:

$$
q_{1}=-\frac{p+p_{2}}{p-p_{2}} q_{2}
$$

and the equality (10.9) is satisfied. To check the identity (10.9) ina generic case, one can use a program of analytical calculation on a computer. However, we have to repeat that these calculations (they were fulfilled once) are not necessary. The identity (10.5) is just the consequence of Theorem 9.4 and the fact of existense of the "extra integral" $I_{5}$. 


\section{Effective Hamiltonian on Water of Finite Depth}

Let us return to the canonical transformation (5.9). In new variables, $\beta_{k}$, we have:

$$
\begin{aligned}
& H=H_{0}+H_{2}+H_{3}+\cdots, \\
& H_{0}=\int \omega_{k}\left|b_{k}\right|^{2} d k, \\
& H_{2}=\frac{1}{4} \int T\left(k, k_{1}, k_{2}, k_{3}\right) b_{k}^{*} b_{k_{1}}^{*} b_{k_{2}} b_{k_{3}} \delta\left(k+k_{1}-k_{2}-k_{3}\right) d k d k_{1} d k_{2} d k_{3}, \\
& H_{3}=\ldots
\end{aligned}
$$

where

$T\left(k, k_{1}, k_{2}, k_{3}\right)=\frac{1}{4}\left(\tilde{T}\left(k, k_{1}, k_{2}, k_{3}\right)+\tilde{T}\left(k_{1}, k, k_{2}, k_{3}\right)+\tilde{T}\left(k_{2}, k_{3}, k, k_{1}\right)+\tilde{T}\left(k_{3}, k_{2}, k, k_{1}\right)\right)$

$\tilde{T}\left(k, k_{1}, k_{2}, k_{3}\right)=V^{(2,2)}\left(k, k_{1}, k_{2}, k_{3}\right)+R^{(1)}\left(k, k_{1}, k_{2}, k_{3}\right)+R^{(2)}\left(k, k_{1}, k_{2}, k_{3}\right)$

and

$$
\begin{aligned}
& R^{(1)}\left(k, k_{1}, k_{2}, k_{3}\right)=-\frac{V^{(0,3)}\left(-k-k_{1}, k, k_{1}\right) V\left(-k_{2}-k_{3}, k_{2}, k_{3}\right)}{\omega\left(-k-k_{1}\right)+\omega(k)+\omega\left(k_{2}\right)} \\
& R^{(2)}\left(k, k_{1}, k_{2}, k_{3}\right)=-\frac{V^{(1,2)}\left(k+k_{1}, k, k_{1}\right) V^{(1,2)}\left(k_{2}+k_{3}, k_{2}, k_{3}\right)}{\omega_{k+k_{1}}-\omega_{k}-\omega_{k_{1}}}- \\
& -\frac{V^{(1,2)}\left(k, k_{2}, k-k_{2}\right) V^{(2,2)}\left(k_{3}, k_{3}-k_{1}, k_{1}\right)}{\omega_{k-k_{2}}-\omega_{k}+\omega_{k_{2}}}-\frac{V^{(1,2)}\left(k, k_{3}, k-k_{3}\right) V^{(1,2)}\left(k_{2}, k_{2}-k_{1}, k_{1}\right)}{\omega_{k-k_{3}}-\omega_{k}+\omega_{k_{3}}}- \\
& -\frac{V^{(1,2)}\left(k_{2}, k, k_{2}-k\right) V^{(1,2)}\left(k_{1}, k_{1}-k_{3}, k_{3}\right)}{\omega_{k_{2}-k}+\omega_{k}-\omega_{k_{2}}}+\frac{V^{(1,2)}\left(k_{3}, k, k_{3}-k\right) V^{(1,2)}\left(k_{2}, k_{2}-k_{1}, k_{1}\right)}{\omega_{k_{3}-k}+\omega_{k}-\omega_{k_{3}}}
\end{aligned}
$$

The motion equation in new variables (5.4) takes the form:

$$
\frac{\partial b_{k}}{\partial y}+i \omega_{k} b_{k}=-\frac{i}{2} \int T\left(k, k_{1}, k_{2}, k_{3}\right) b_{k_{1}}^{*} b_{k_{2}} b_{k_{3}} \delta\left(k+k_{1}-k_{2}-k_{3}\right) d k_{1} d k_{2} d k_{3}
$$

Mostly this equation has to be considered on the resonant manifold, describing four-wave interactions:

$$
\begin{aligned}
& \omega_{k}+\omega_{k_{1}}=\omega_{k_{2}}+\omega_{k_{3}} \\
& k+k_{1}=k_{2}+k_{3}
\end{aligned}
$$


In the limit of deep water equation (11.7) coincides on this resonant manifold with the equation derived by the author in 1968 (V.Zakharov, 1968).

Suppose, that all $k_{i}$ in $T\left(k, k_{1}, k_{2}, k_{3}\right)$ are of the same order $k$. On deep water in the limit

$$
k h \rightarrow \infty
$$

all the terms in (11.4) are comparable and

$$
T\left(k, k_{1}, k_{2}, k_{3}\right) \simeq k^{3}
$$

On shallow water in the limit

$$
k h \rightarrow 0
$$

we have to study the behavior of all three components of $\tilde{T}\left(k, k_{1}, k_{2}, k_{3}\right)$ separately. According to (8.6), the first term in (11.4) behaves like $k h \times k^{3}$, and can be neglected. Apparently,

$$
R^{(1)}\left(k, k_{1}, k_{2}, k_{3}\right) \simeq \frac{k^{3}}{k h} \simeq \frac{k^{2}}{h} .
$$

The most interesting term in (11.4) is $R^{(2)}\left(k, k_{1}, k_{2}, k_{3}\right)$. Let us consider it on a resonant manifold (11.8). If vectors $k_{1}, k$ are far from being parallel, denominators in (11.6) are not small, and we have the estimate:

$$
R^{(2)}\left(k, k_{1}, k_{2}, k_{3}\right) \simeq \frac{k^{2}}{h}
$$

However, if $k_{1}$ and $k$ are almost parallel, in virtue of (11.8), $k_{2}$ and $k_{3}$ are almost parallel to $k$ as well. In this case all the denominators in (11.6) are small. They reach their minimal values if all the vectors $k_{i}$ are parallel. For instance, for parallel vectors we have:

$$
\omega_{k+k_{1}}-\omega_{k}-\omega_{k_{1}}=c\left(k+k_{1}-k-k_{1}\right)-\alpha\left(k+k_{1}\right)^{3}+\alpha k^{3}+\alpha k_{1}^{3}=3 \alpha k k_{1}\left(k+k_{1}\right)
$$

and the first term in (11.6) is equal:

$$
\frac{V^{1,2}\left(k, k_{1}, k+k_{1}\right) V^{1,2}\left(k_{2}+k_{3}, k_{2}, k_{3}\right)}{\omega_{k+k_{1}}-\omega_{k}-\omega_{k_{1}}} \simeq \frac{9 g^{1 / 2}}{16 \pi^{2} h^{3}} .
$$

The following fact is the direct consequence of "formal integrability" of KP-2 equation:

The terms of the order $1 / h^{3}$ on the resonant manifold (11.8) are cancelled.

Indeed, $R^{(2)}\left(k, k_{1}, k_{2}, k_{3}\right)$ reaches its maximal value when all the vectors $k_{i}$ are almost parallel. Suppose that $k$ is a given vector and introduce on planes $k_{i}(i=1,2,3)$ coordinates $\left(p_{i}, q_{i}\right)$, where $p_{i}$ are parallel to $k$ while $q_{i}$ are orthogonal to $k$. According to our assumptions all $p_{i}$ are positive and $\left|q_{i}\right| \ll\left|p_{i}\right|$. Expanding $\omega(p, q)$ in Taylor series, we can rewrite the resonant conditions (11.8) as follow: 


$$
\begin{aligned}
& q_{1}=q_{2}+q_{3} \\
& p+p_{1}=p_{2}+p_{3}, \\
& -\alpha\left(p^{3}+p_{1}^{3}\right)+\frac{1}{2} \frac{q_{1}^{2}}{p_{1}}=-\alpha\left(p_{2}^{3}+p_{3}^{3}\right)+\frac{1}{2} \frac{q_{2}^{2}}{p_{2}}+\frac{1}{2} \frac{q_{3}^{3}}{p_{3}} .
\end{aligned}
$$

In the considered case $\alpha>0$ and these conditions can be transformed to the equations (10.6)-(10.8) by a trivial change of variables. As far as all the vectors $k_{i}$ are almost parallel, one can use for $V^{(1,2)}\left(k, k_{1}, k_{2}\right)$ its simplified expression (8.9). As a result, the leading term in $R^{(2)}\left(k, k_{1}, k_{2}, k_{3}\right)$ is proportional to $T^{-1,-1,1,1}\left(k, k_{1}, k_{2}, k_{3}\right)$ given by (9.14). According to Theorem 9.1, it is zero. In the particular case $p_{2}<p, p_{3}<p$, the leading term in $R^{(2)}\left(k, k_{1}, k_{2}, k_{3}\right)$ coincides with (11.4).

Cancellation of the most singular terms in $T\left(k, k_{1}, k_{2}, k_{3}\right)$ on the resonant manifold (11.8) is an extremely nontrivial fact, which hardly could be discovered unless a proper development of the Inverse Scattering Method is done. The fact of cancellation makes the problem of numerical simulation of surface waves on shallow water much more hard and tricky than on deep water.

There is one more posiibility to make denominators in $R^{(2)}\left(k, k_{1}, k_{2}, k_{3}\right)$. The resonant conditions (11.8) can not be satisfied if one of the vectors $k_{i}$ is zero. However, in the limit $k h \rightarrow 0$ these conditions could be satisfied if the length of one of the vectors, suppose $k_{3}$, is much less than the length of other vectors, which in this case must be almost parallel.

Let us consider this situation on the simpliest example. Suppose, vectors $k, k_{1}, k_{2}$ are of the same length, parallel, and pointed in the same direction, while vector $k_{3}$ is small, parallel to $k$, but pointed in the opposite direction. Hence $k_{i}=p_{i}(i=0,1,2)$ and $k_{3}=-p_{3}$. We have now:

$$
\begin{aligned}
& p+p_{1}=p_{2}-p_{3} \\
& \omega(p)+\omega\left(p_{1}\right)=\omega\left(p_{2}\right)+\omega\left(p_{3}\right) .
\end{aligned}
$$

Taking $\omega \simeq S\left(p-\alpha p^{3}\right)$, one can find from (11.15):

$$
p_{3} \simeq 3 \alpha p p_{1}\left(p+p_{1}\right) .
$$

For pure gravity waves we have the estimate:

$$
\frac{R_{3}}{k} \simeq(k h)^{2}
$$

and the denominator

$$
\omega_{k+k_{1}}-\omega_{k}-\omega_{k_{1}} \simeq \omega_{k}(k h)^{2} .
$$

The same estimate is correct if vectors $k, k_{1}, k_{2}$ are slightly nonparallel. The small vector $k_{3}$ in this case can be pointed in an arbitrary direction. The estimate (11.16) shows that 
the process under discussion is "quasi-resonant". As far as $k_{3}$ is not parallel to $k_{i}$, there is no reason for cancellation of this type terms in $R^{(2)}\left(k, k_{1}, k_{2}, k_{3}\right)$. Due to the factor $\left|k_{3}\right|^{1 / 2}$ in the coefficients $V\left(k, k_{1}, k_{2}\right)$, the final estimate for the coefficient of four-wave quasi-resonant processes including a long wave is:

$$
T \simeq \frac{1}{h^{3}} k h \simeq \frac{k}{h^{2}}
$$

A possible significance of the processes of this type was mentioned by Lin and Perrie (Lin,Perrie, 1997). To estimate a real importance of these processes one has to take into account their small "phase volume". Indeed, $k_{3}^{2} \simeq k^{2}(k h)^{4}$ is a very small factor. Thus, the contribution of quasi-resonant processes depends essentially of the shape of distribution of energy in the $k$-space. This is a subject of a special detailed considiration.

Cancellation of the most important terms in the coefficient of four-wave interaction can lead to a hypothesis that some high-order (five-waves, six-waves, etc.)interactions could be more important on shallow water than four-wave interaction. This is not true. In virtue of Theorem 9.2 the leading terms in the coefficient of five-wave interaction are cancelled, as well as in the coefficient of four-wave interaction. The same is correct in any order. According to Theorem 9.2, in KP-2 equation coefficients of all $n$-wave interactions on the corresponding resonant manifolds are zero. In a real situation they are non-zero. However they are just "remnant", survived after cancellation of the leading terms, caused by integrability of KP-2 equation. The accurate calculation of these coefficients is a hard and time-consuming problem.

\section{Non-Hamiltonian Effects}

So far we discussed only wave-wave interaction described by the Hamiltonian fluid dynamics. But the system of surface waves is not closed in a physical sense and certain non-Hamiltonian effects are very important for developing of an adequate models of winddriven sea waving. The most important among those effects is "wave-breaking" normally providing dissipation of most of the wave energy. An initial stage of the wave breaking can be described in tyhe framework of Hamiltonian dynamics. It is the formation of a singularity on the initially smooth surface of a fluid.

Elaboration of a reliable theory of this process is one of the most challenging problems in Nonlinear Mathematical Physics. The most probable scenario of wave-breaking is the formation, in a finite time, of a "wedge-type" singularity - discontinuity of first space derivative $\eta_{x}$.

However, it is only the first act of wave-breaking scenario. The second act is the formation of a "white cap" - a surface covered with foam from above and full of intense turbulence and air bubbles inside. At most, one can hope to develop a proper qualitative and phenomenological theory of these types of phenomena. But in a typical situation wave-breaking is rare and the part of sea surface covered with "white caps" is small. 
In this case wave breaking phenomena can be treated as a "universal sink" of wave energy in the region of large wave numbers and taken into account just as the boundary condition imposed at $k \rightarrow \infty$. The whole theory can be developed without the knowledge of the detailed mechanism of wave-breaking and the connected phenomena.

Another fundamental non-Hamiltonian process is the wind-wave interaction. This interaction is actually nothing but Cherenkov-type instability causing the generation of all waves having a small enough phase velocity, less than the wind velocity at a certain standard height. In oceanography this instability is traditionally called "Miles instability" (see Miles, 1958). Analytical calculations of the growth rate of this instability is a very hard problem due to intensive turbulence of the wind. Nevertheless, this growth rate is known from numerous experiments and computer simulations.

Due to the presence of the small parameter $\epsilon=\rho_{a} / \rho_{w}\left(\rho_{a}, \rho_{w}\right.$ are air and water densities correspondingly), the interaction with a wind is weak, and one can take it into account just by replacing in (11.7)

$$
\omega_{k} \rightarrow \omega_{k}+i \beta_{k}
$$

For $\beta_{k}$ one can accept the expression offered by M.Donelan (M.Donelan, 1993):

$$
\begin{aligned}
& \beta_{k}=0,194 \epsilon \omega_{k}\left(\frac{\vec{u} \vec{k}}{\omega_{k}}-1\right)^{2} \quad \text { if } \quad \frac{\vec{u} \vec{k}}{\omega_{k}}-1>0 \\
& \beta_{k}=0 \quad \text { if } \quad \frac{\vec{u} \vec{k}}{\omega_{k}}-1<0
\end{aligned}
$$

This simple expression is especially good for short waves, $\vec{u} \vec{k} \gg \omega_{k}$.

On a finite depth another non-Hamiltonian effect has to be taken into account. This is the bottom friction, which could not be excluded from consideration for two reasons:

1. Bottom friction is the mechanism of wave energy dissipation. $I_{N}$ can be taken into account by change in (11.7):

$$
\omega_{k} \rightarrow \omega_{k}-i \gamma_{k}
$$

According to S.Weber (Komen et al, 1994):

$$
\gamma_{k}=c_{f} \frac{|k|}{\sinh 2 k h}
$$

where $c_{f}$ typically is equal $0.001-0.01 \mathrm{~m} / \mathrm{sec}$, depending on the bottom and flow conditions. It is important that $\gamma_{k}$ decays exponentially at $k h \rightarrow \infty$ and turns to

$$
\gamma_{k} \simeq \frac{c_{f}}{2 h}
$$

if $k h \rightarrow 0$. 
2. Bottom friction leads to the additional mechanism of nonlinear wave interaction. Cubic term $H_{1}$ in the Hamiltonian (3.15) and the corresponding quadratic terms in the motion equation (4.5) generates forced beating with wave numbers $k_{1} \pm k_{2}$, and frequencies $\omega_{k_{1}} \pm \omega_{k_{2}}$. These beatings, like the initial wave, loose the energy due to the bottom friction.

A linear dissipating of the beating is an additional reason for the nonlinear interaction of the basic waves. This is essentially non-Hamiltonian process. Similar processes have been studied very well in the theory of plasma turbulence (see, for instance, Zakharov, Musher, Rubenchik, 1985).

It is remarcable that this non-Hamiltonian process can be still described in a framework of equation (11.7). We just have to modify;

$$
T\left(k, k_{1}, k_{2}, k_{3}\right) \rightarrow T\left(k, k_{1}, k_{2}, k_{3}\right)+i S\left(k, k_{1}, k_{2}, k_{3}\right),
$$

where $T\left(k, k_{1}, k_{2}, k_{3}\right)$ is the Hamiltonian inyteraction coefficient satisfying the condition:

$$
T\left(k, k_{1}, k_{2}, k_{3}\right)=T^{*}\left(k, k_{1}, k_{2}, k_{3}\right)=T\left(k_{1}, k, k_{2}, k_{3}\right)=T\left(k_{2}, k_{3}, k, k_{1}\right)
$$

The non-Hamiltonian term $S\left(k, k_{1}, k_{2}, k_{3}\right)$ can be calculated by using of methods of multiscale expansions, implemented in the article (V.Zakharov, 1968). Omitting the details of this calculation, let us present the final result:

$$
\begin{aligned}
& S\left(k, k_{1}, k_{2}, k_{3}\right)=\gamma_{k-k_{2}} \frac{V^{(1,2)}\left(k, k_{2}, k-k_{2}\right) V^{(1,2)}\left(k_{3}, k_{3}-k_{1}, k_{1}\right)}{\left(\omega_{k-k_{2}}-\omega_{k}+\omega_{k_{2}}\right)^{2}}+ \\
& +\gamma_{k-k_{3}} \frac{V^{(1,2)}\left(k, k_{3}, k-k_{3}\right) V^{(1,2)}\left(k_{2}, k_{2}-k_{1}, k_{1}\right)}{\left(\omega_{k-k_{3}}-\omega_{k}+\omega_{k_{3}}\right)^{2}}- \\
& -\gamma_{k_{1}-k_{2}} \frac{V^{(1,2)}\left(k_{1}, k_{2}, k_{1}-k_{2}\right) V^{(1,2)}\left(k_{3}, k_{3}-k, k\right)}{\left(\omega_{k_{1}-k_{2}}-\omega_{k}+\omega_{k_{2}}\right)^{2}}- \\
& -\gamma_{k_{1}-k_{3}} \frac{V^{(1,2)}\left(k_{1}, k_{3}, k_{1}-k_{3}\right) V^{(1,2)}\left(k_{2}, k_{2}-k, k\right)}{\left(\omega_{k_{1}-k_{3}}-\omega_{k_{1}}+\omega_{k_{3}}\right)^{2}}
\end{aligned}
$$

The expression (12.8) satisfies the following symmetry condition:

$$
S\left(k, k_{1}, k_{2}, k_{3}\right)=S\left(k, k_{1}, k_{2}, k_{3}\right)=-S\left(k, k_{1}, k_{2}, k_{3}\right)
$$

In virtue of this condition the equation (11.7), though non-Hamiltonian still preserve the constant of motion, the total wave action:

$$
\frac{d N}{d t}=0, \quad N=\int\left|b_{k}\right|^{2} d k
$$

if only $G_{m} \omega_{k}=0$.

Equation (11.7) includes all non-Hamiltonian corrections and is a proper and convinient base for statistical description of small-amplitude waves on a surface of a finite-depth fluid. 


\section{References}

- G.B.Airy, Tides and Waves, On the Theory of Oscillatory Waves, Camb.Trans., 8, (1847), 441-473.

- D.Chalikov, The Numerical Simulation of Wind-Wave Interaction, J.Fluid Mech., 87 (3), (1978), 561-582.

- D.Crawford, H.Yuen, P.Saffman, Evolution of a Random Inhomogenous Field of Nonlinear Deep Water Gravity Waves, J. Wave Motion, (1980), 1-16.

- W.Craig, M.Groves, Hamiltonian Long-Wave Approximations of the Water-Wave problem, Wave Motion, 19, (1994), 376-389.

- W.Craig, S.Sulem, Numerical Simulation of Gravity Waves, J. of Computational Physics, 108 (1), (1993), 73-83.

- M.Donelan, Air-Water Exchange Processes, Proceedings of the IUTAM Symposium on Physical Limnology, 10-14 September, 1993, Broome, Australia,

- V.Dryuma, Analytic Solution of the Two-Dimensional Korteveg - de Vries Equation, Soviet JETP Lett, 19, (1974), 387-389.

- A.Dyachenko, Yu.Lvov, V.Zakharov, Five-wave interaction on the surface of deep fluid Physica D, 87 (1995), 233-261.

- A.Dyachenko, Yu.Lvov, On the Hasselmann's and Zakharov's approaches to kinetic equations for the gravity waves, J.Phys.Ocean., 1996

- A.Dyachenko, E.Kuznetzov, M.Spector, V.Zakharov, Analytic description of freesurface dynamics of a deep fluid, Phys.Lett.A, 221, (1996), 73

- K.Hasselmann, On the nonlinear energy transfer in gravity-wave spectrum, J. Fluid Mech., 12, (1962), 481-500.

- B.Kadomtsev, Plasma Turbulence, Academic Press, New York, 1965.

- G.Komen, L.Cavaleri, M.Donelan, S. Hasselmann, K. Hasselmann, P. Janssen, Dynamics and modelling of ocean waves, Cambridge University Press, 1994.

- B.Konopelchenko, Solitons in Multidimensions. Inverse Spectral Transform Method, World Scientific Publishing Co., Singapore, 1993.

- I.Krichever, Spectral Theory of Two-Dimensional Periodic Operators, Uspekh.Mat.Nauk, (1989), 121-184. 
- V. Krasitskii, On the canonical transformation of the theory of weakly nonlinear waves with non-decay dispersion law, Sov. Phys. JETP, 98,(1990), 1644-1655.

- E. Kuznetzov, M. Spector, V. Zakharov, Surface singularities of ideal fluid, Phys. Lett. A, 182, (1993), 387-393.

- E.Kuznetzov, M.Spector, V.Zakharov, Formation of singularities on the free surface of an ideal fluid, Phys.Rev.E, 49 (2), (1994),

- Q.-R. Lin, W.Perrie, A new coastal wave model. Part 3. Nonlinear wave-wave interaction, J.Phys.Oceanogr. (1997), in press.

- J.Miles, On the Generation of Surface Waves by Shear Flow, J.Fluid Mech., 3, (1957), 185-204.

- O.Phillips, Spectral and statistical properties of the equilibrium range in windgenerated gravity waves, J.Fluid Mech., 156, (1985), 505-531.

- A.Radder, An Explicit Hamiltonian Formulation of Surface Waves in Water of Finite Depth, J,Fluid Mech., 237, (1992), 435-455.

- E.Shulman, Teor.Mat.Fiz., 76, (1988), 88-89.

- G.Stokes, On the Theory of Oscillatory Waves, Camb.Trans., 8, (1947), 441-473.

- V.Zakharov, The Wave Stability in Nonlinear Media, Sov.Phys.JETP, 24, (1967), 455-459.

- V.Zakharov, Stability of periodic waves of finite amplitude on a surface of a deep fluid, J.Appl.Mech.Tech.Phys., 2, (1968), 190-198.

- V. Zakharov, Inverse and direct cascade in the wind-driven surface wave turbulence and wave-breaking, in "Breaking waves", M. Banner, H. Grimshaw (Eds), IUTAM symposium, Sydney, Australia, Springer - Verlag, (1992), 69-91.

- V.Zakharov, A.Balk, E.Shulman, Conservation and Scattering in Nonlinear Wave Systems, in "Important development in Soliton Theory", Ed. A.Fokas, V.Zakharov, Springer-Verlag, (1993), 375-403.

- V.Zakharov, G.Falkovich, V.Lvov, Kolmogorov spectra of wave turbulence, SpringerVerlag, Heidelberg - Berlin, 1992.

- V.Zakharov, N.Filonenko, The energy spectrum for stochastic oscillation of a fluid's surface, Doklady Akad.Nauk, 170, (1966), 1292-1295.

- V.Zakharov, V.Kharitonov, Instability of Monochromatic Waves on the Surface of an Arbitrary Depth Fluid, Prikl.Mekh.Tekh.Fiz., 5, (1970), 45-50. 
- V.Zakharov, E.Kuznetsov, Hamiltonian Formalism for System of Hydrodynamic type, Math.Phys.Rev., 4, (1984), 167-220.

- V.Zakharov, S.Musher, A.Rubenchick, Hamiltonian Approach in Description of Nonlinear Plasma Phenomena, Phys.Reports, 129, (1985), 285-366.

- V.Zakharov, E.Shulman, Degenerative Dispersion Laws, Motion Invariants and Kinetic Equation, Physica D, (1980), 192-202.

- V.Zakharov, E.Shulman, Integrability of nonlinear waves and Perturbation Theory, in "What is integrability?", Ed. V.Zakharov, Springer-Verlag, 1991.

- V.Zakharov, E.Shulman, On Additional Motion Integrals of Nonlinear Wave Systems, Physica D, 29 (1988), 283-320. 